\title{
On the Information Loss of the Max-Log Approximation in BICM Systems
}

\author{
Mikhail Ivanov, Christian Häger, Fredrik Brännström, Alexandre Graell i Amat, Alex Alvarado, and Erik Agrell
}

\begin{abstract}
We present a comprehensive study of the information rate loss of the max-log approximation for $M$-ary pulse-amplitude modulation (PAM) in a bit-interleaved coded modulation (BICM) system. It is widely assumed that the calculation of L-values using the max-log approximation leads to an information loss. We prove that this assumption is correct for all $M$-PAM constellations and labelings with the exception of a symmetric 4-PAM constellation labeled with a Gray code. We also show that for max-log L-values, the BICM generalized mutual information (GMI), which is an achievable rate for a standard BICM decoder, is too pessimistic. In particular, it is proved that the so-called "harmonized" GMI, which can be seen as the sum of bit-level GMIs, is achievable without any modifications to the decoder. We then study how bit-level channel symmetrization and mixing affect the mutual information (MI) and the GMI for max-log L-values. Our results show that these operations, which are often used when analyzing BICM systems, preserve the GMI. However, this is not necessarily the case when the MI is considered. Necessary and sufficient conditions under which these operations preserve the MI are provided.
\end{abstract}

Index Terms-Bit-interleaved coded modulation, generalized mutual information, logarithmic likelihood ratio, max-log approximation, mismatched decoder.

\section{INTRODUCTION}

Bit-interleaved coded modulation (BICM) [1]-[3] is a pragmatic approach to achieve high spectral efficiency with binary error-correcting codes. Because of its inherent simplicity and flexibility, as well as good performance, it is implemented in many practical wireless communication systems [4]-[6].

A central part of a BICM system is the demapper, which computes soft information about the coded bits in the form of so-called L-values. Ideally, L-values correspond to $\log$ likelihood ratios, in which case we refer to them as exact L-values. In practice, however, the demapper often computes only approximate L-values due to complexity reasons. A common approximation is to replace the log-sum operation in the log-likelihood ratio computation with a max-log operation. This approximation can be motivated by the fact that at high signal-to-noise ratio (SNR), exact and approximate max-log L-values are almost identical.

This research was supported by the Swedish Research Council, Sweden, under Grant No. 2011-5950, the Ericsson's Research Foundation, Sweden, and the European Community's Seventh's Framework Programme (FP7/20072013) under grant agreement No. 271986.

M. Ivanov, C. Häger, F. Brännström, A. Graell i Amat, and E. Agrell are with the Dept. of Signals and Systems, Chalmers Univ. of Technology, SE-41296 Gothenburg, Sweden (e-mail: \{mikhail.ivanov, christian.haeger, fredrik.brannstrom, alexandre.graell, agrell\}@ chalmers.se).

A. Alvarado is with the Optical Networks Group, Dept. of Electronic \& Electrical Engineering, Univ. College London, WC1E 7JE London, UK (email: alex.alvarado@ieee.org).
In this paper, we analyze achievable rates of BICM for nonbinary pulse-amplitude modulation (PAM) constellations over the additive white Gaussian noise (AWGN) channel, paying special attention to max-log L-values. Traditionally, achievable rates for BICM systems are analyzed for exact Lvalues under the assumption of an ideal interleaver [7], which results in the BICM mutual information (MI) (i.e., the sum of $m$ bit-level MIs), often referred to as the BICM capacity. In [8], it was proposed to analyze BICM from a mismatched decoding perspective, showing that the maximum achievable rate for a BICM system is lowerbounded by the BICM generalized mutual information (GMI), without invoking any interleaver assumption. For exact L-values, the BICM GMI coincides with the BICM MI [8].

When max-log L-values are considered, most of the previous work concentrates on the correction of the "suboptimal" L-values in order to either maximize the BICM GMI [9], [10] or minimize the error probability [11]. To the best of our knowledge, a rigorous comparison of achievable rates in terms of the BICM MI and the BICM GMI for max-log L-values has not yet been carried out. Despite this fact, it seems to be a common belief in the literature that the calculation of max$\log \mathrm{L}$-values is inherently an information lossy operation. As an example, when discussing the MI between the transmitted information symbol and the vector of max-log L-values at the output of the demapper, [12, p. 137] concludes that "the approximation clearly constitutes a lossy procedure and entails an inferior BICM capacity”. Similar implicit assumptions are made in [13] and [14]. We prove that this conclusion is not always true. In particular, we prove that for symmetric 4PAM constellations labeled with the binary reflected Gray code (BRGC), no information loss occurs when comparing exact and max-log L-values, i.e., the BICM MI is the same in both cases. We also prove that for all other combinations of PAM constellations and labelings, the max-log approximation indeed induces an information loss.

We then study the BICM GMI for max-log L-values. In particular, the so-called "harmonized" GMI was introduced in [10] as an achievable rate for a modified BICM decoder that applies scaling factors to the L-values. In this paper, we argue that the L-value scaling is in fact unnecessary, and the harmonized GMI (which can be seen as the sum of $m$ bit-level GMIs) is achievable without any modifications to the decoder. Finally, we analyze two common processing techniques which are often used in the theoretical analysis of BICM systems: bitlevel channel symmetrization and channel mixing. The results show that these operations do not affect the BICM GMI but can reduce the BICM MI. 


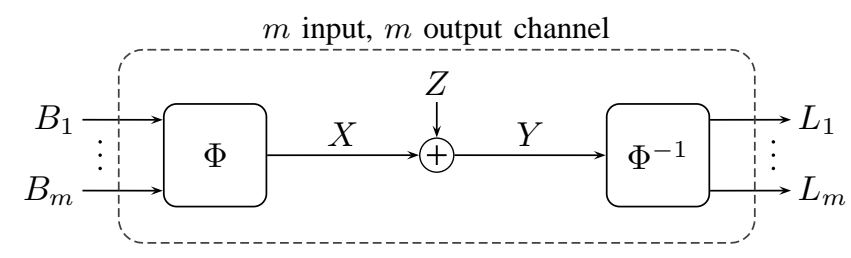

Fig. 1: Block diagram of the analyzed system.

The results presented in this paper can be easily generalized to multi-dimensional product constellations of $M$-PAM of not necessarily the same size labeled with a product labeling [15. Sec. X].

\section{A. Notation}

Throughout the paper, boldface letters $\boldsymbol{x}$ denote row vectors, blackboard letters $\mathbb{X}$ denote matrices, and capital letters $X$ denote random variables (RVs). $\mathbf{1}_{n}$ and $\mathbf{0}_{n}$ denote all-one and all-zero vectors of length $n$, respectively. Calligraphic letters $\mathcal{X}$ denote sets, where $\mathbb{R}$ stands for the set of real numbers and $\mathbb{N}$ for the set of natural numbers. For $n \in \mathbb{N}$, we define $[n]=\{1,2, \ldots, n\}$. We define $\mathcal{B}=\{0,1\}$. If $b \in \mathcal{B}$, then $\bar{b}=(-1)^{(b+1)} \in\{-1,+1\}$ and $\bar{b}=1-b$. E $\{\cdot\}$ denotes expectation and $\operatorname{Pr}(\cdot)$ represents probability. The probability density function (PDF) of a continuous RV $Y$ is denoted by $f_{Y}(\cdot)$ and the conditional PDF by $f_{Y \mid X}(\cdot \mid \cdot)$. The probability mass function (PMF) of a discrete $\mathrm{RV} X$ is denoted by $p_{X}(\cdot)$.

\section{System MODEL}

A block diagram of the considered system model, which we discuss in the following, is shown in Fig. 1.

\section{A. Modulator}

A modulator $\Phi$ is fed with $m$ bits $B_{j}, j \in[m]$, and maps them to one of $M=2^{m}$ possible constellation points. We consider one-dimensional PAM constellations denoted by $\mathcal{S}=\left\{a_{1}, \ldots, a_{M}\right\}$, where $a_{1}<\cdots<a_{M}$. We say that the constellation is symmetric (around $y_{0}$ ) if $a_{k}=-a_{M-k+1}+$ $2 y_{0}$ for $k \in[M]$ and some $y_{0} \in \mathbb{R}$, and we say that the constellation is equally spaced if $a_{k+1}-a_{k}$ is independent of $k$. The bits are assumed to be independent and distributed according to $p_{B_{j}}(u)=1 / 2, \forall j$ and $u \in \mathcal{B}$. Thus, the symbols are equiprobable, i.e., $p_{X}\left(a_{k}\right)=1 / M, \forall k \in[M]$. The constellation is assumed to be normalized to unit average energy $\mathrm{E}\left\{X^{2}\right\}=(1 / M) \sum_{k=1}^{M} a_{k}^{2}=1$.

The mapping $\{0,1\}^{m} \rightarrow \mathcal{S}$ performed by the modulator is assumed to be one-to-one and is defined by a binary labeling. The binary labeling is specified by an $m \times M$ binary matrix $\mathbb{L}$, where the $k$ th column of $\mathbb{L}$ is the binary label of the constellation point $a_{k}$. Furthermore, we define $\mathcal{S}_{j, u}=\left\{a_{k} \in \mathcal{S}: \mathbb{L}_{j, k}=u, \forall k \in[M]\right\}$ as the subconstellation consisting of all points labeled with the bit $u$ in the $j$ th bit position.

Certain quantities, such as the L-values we define below, depend only on the subconstellations $\mathcal{S}_{j, 0}$ and $\mathcal{S}_{j, 1}$, i.e., they depend only on the $j$ th row in $\mathbb{L}$. We refer to the $j$ th row of $\mathbb{L}$ as a bit pattern, or simply pattern, which was shown in [16]

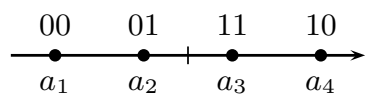

(a) 4-PAM with BRGC.

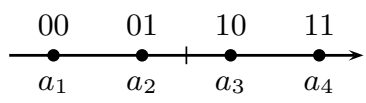

(b) 4-PAM with NBC.

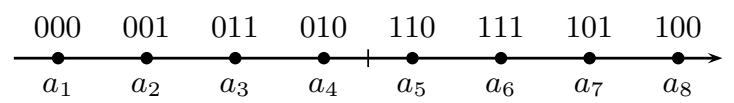

(c) 8-PAM with BRGC

Fig. 2: Examples of equally spaced PAM constellations with different binary labelings.

to be a useful tool for analyzing binary labelings. A pattern is defined as a vector $\boldsymbol{p}_{j}=\left[p_{1}, \ldots, p_{M}\right] \in \mathcal{B}^{M}$ with Hamming weight $M / 2$. A labeling $\mathbb{L}$ can then be represented by $m$ different patterns, each corresponding to one row of $\mathbb{L}$. We define two trivial operations that can be applied to a pattern. A reflection of $\boldsymbol{p}$ is defined as $\boldsymbol{p}^{\prime}=\operatorname{refl}(\boldsymbol{p})$ with $p_{k}^{\prime}=p_{M+1-k}$. An inversion of $\boldsymbol{p}$ is defined as $\boldsymbol{p}^{\prime}=\operatorname{inv}(\boldsymbol{p})$ with $p_{k}^{\prime}=\bar{p}_{k}$. We say that a pattern is symmetric if $\boldsymbol{p}=\operatorname{refl}(\boldsymbol{p})$. A pattern $\boldsymbol{p}^{\prime}$ that is related to another pattern $\boldsymbol{p}$ via inversions and/or reflections is said to be equivalent to $\boldsymbol{p}$. Analogously, labelings related by trivial operations (i.e., row permutations, inversion, and/or reflection of all patterns in the labeling) are said to be equivalent [15, Definition 6b]. For example, there exist eight labelings that are equivalent to the BRGC for 4-PAM shown in Fig.2(a). For symmetric constellations, equivalent patterns and labelings behave similarly, e.g., they give the same uncoded bit error rate (BER) and achievable rates.

Most of the numerical examples are presented for an equally spaced 4-PAM constellation, shown in Fig. 2[a),(b), together with the two labelings

$$
\mathbb{L}_{1}=\left[\begin{array}{llll}
0 & 0 & 1 & 1 \\
0 & 1 & 1 & 0
\end{array}\right], \quad \mathbb{L}_{2}=\left[\begin{array}{llll}
0 & 0 & 1 & 1 \\
0 & 1 & 0 & 1
\end{array}\right]
$$

which are often referred to as the BRGC and the natural binary code (NBC) or set-partitioning labeling [17], respectively. An example of an equally spaced 8-PAM constellation labeled with the BRGC is shown in Fig. 2](c).

\section{B. AWGN Channel}

The constellation points are assumed to be transmitted over the discrete-time memoryless AWGN channel with output $Y=X+Z$, where the noise $Z$ is a zero-mean Gaussian $\mathrm{RV}$ with variance $\mathrm{E}\left\{Z^{2}\right\}=N_{0} / 2$. The conditional PDF of the channel output given the channel input is

$$
f_{Y \mid X}(y \mid x)=\sqrt{\frac{\rho}{\pi}} e^{-\rho(y-x)^{2}}
$$

where $\rho=1 / N_{0}$ is the average SNR.

\section{Demappers and L-values}

Two demappers $\Phi^{-1}$ are considered at the receiver. The first one calculates exact L-values as the log-likelihood ratios

$$
l_{j}^{\mathrm{ex}}(y)=\log \frac{f_{Y \mid B_{j}}(y \mid 1)}{f_{Y \mid B_{j}}(y \mid 0)}=\log \frac{\sum_{x \in \mathcal{S}_{j, 1}} e^{-\rho(y-x)^{2}}}{\sum_{x \in \mathcal{S}_{j, 0}} e^{-\rho(y-x)^{2}}} .
$$




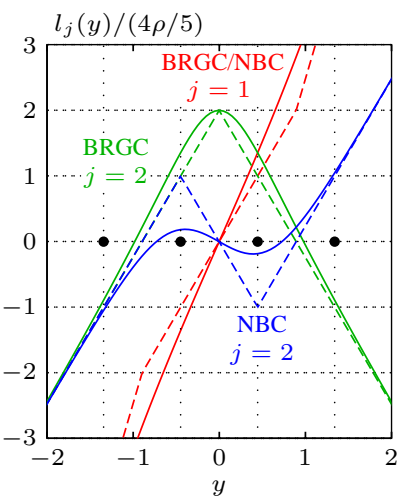

(a) $\rho=0 \mathrm{~dB}$.

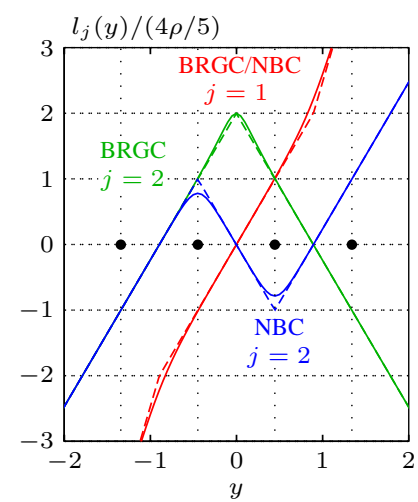

(b) $\rho=6 \mathrm{~dB}$.
Fig. 3: Normalized exact (solid) and max-log (dashed) L-values as functions of the observation $y$ assuming an equally spaced 4-PAM constellation labeled with the BRGC and NBC (see Fig. 2]a)-(b)).

The second demapper calculates max-log L-values using the max-log approximation as [18]

$$
l_{j}^{\mathrm{ml}}(y)=\rho\left[\min _{x \in \mathcal{S}_{j, 0}}(y-x)^{2}-\min _{x \in \mathcal{S}_{j, 1}}(y-x)^{2}\right] .
$$

The observation $Y$ is an RV and thus, so are the L-values. To simplify the notation, we use $L_{j}=L_{j}^{\mathrm{ex}}=l_{j}^{\mathrm{ex}}(Y)$ when discussing exact L-values and $L_{j}=L_{j}^{\mathrm{ml}}=l_{j}^{\mathrm{ml}}(Y)$ when discussing max-log L-values. We further define the vector $\boldsymbol{L}=\left[L_{1}, \ldots, L_{m}\right]$ and write $\boldsymbol{L}^{\mathrm{ex}}$ and $\boldsymbol{L}^{\mathrm{ml}}$ when discussing exact and max-log L-values, respectively.

In Fig. 3, we show an example of the exact and max$\log$ L-values (normalized by $4 \rho / 5$ ) as functions of the observation $y$ for the 4-PAM constellation and labelings shown in Fig. 2(a) (b) and two different values of $\rho$. As shown in [13], the max-log L-value is a piecewise linear function of the observation, which simply scales with SNR, whereas the dependency of the exact L-value on the SNR is nonlinear. However, when the SNR increases, one can show that the exact L-value approaches the max-log L-value, in the sense that $\lim _{\rho \rightarrow \infty} l_{j}^{\text {ex }}(y) / \rho=l_{j}^{\mathrm{ml}}(y) / \rho \forall y \in \mathbb{R}$, where $l_{j}^{\mathrm{ml}}(y) / \rho$ is independent of $\rho$ (see (4)).

From Fig. 3, one can observe that the exact L-value for the second bit position of the BRGC (and also the max-log Lvalue) is an even function assuming an equally spaced 4-PAM constellation. More generally, we have the following result, which will be used later on.

Lemma 1. The exact $L$-value $l_{j}^{\mathrm{ex}}(y)$ is symmetric with respect to $y_{0} \in \mathbb{R}$, i.e., $l_{j}^{\mathrm{ex}}\left(y_{0}+y\right)=l_{j}^{\mathrm{ex}}\left(y_{0}-y\right)$ for $y \in \mathbb{R}$, if and only if the constellation is symmetric around $y_{0}$ and the pattern corresponding to the $j$ th bit position satisfies $\boldsymbol{p}=\operatorname{refl}(\boldsymbol{p})$.

Proof: The proof is given in Appendix A

Remark 1. Since the exact L-value $l^{\mathrm{ex}}(y)$ is not a periodic function, the symmetry point is unique, i.e., there cannot exist two distinct $y_{0}, y_{0}^{\prime} \in \mathbb{R}$ such that both $l^{\mathrm{ex}}\left(y+y_{0}\right)=l^{\mathrm{ex}}(-y+$ $\left.y_{0}\right)$ and $l^{\mathrm{ex}}\left(y+y_{0}^{\prime}\right)=l^{\mathrm{ex}}\left(-y+y_{0}^{\prime}\right)$ hold for all $y \in \mathbb{R}$.

Remark 2. It can be shown that Lemma 1 holds without change also for the max-log L-value.

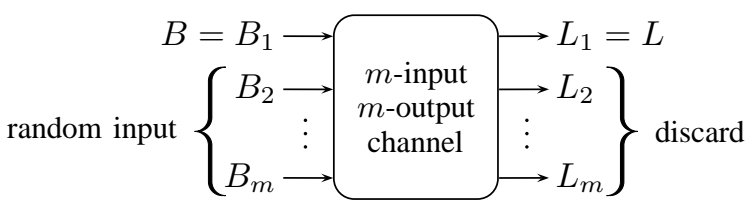

Fig. 4: Bit-level channel, illustrated for the first bit position.

\section{Coding Scheme}

We consider a coding scheme where an information message is mapped to a codeword $\boldsymbol{x}=\left[x_{1}, \ldots, x_{N}\right], x_{i} \in \mathcal{S}$ for $i \in[N]$, and $N$ corresponds to the number of (AWGN) channel uses. The set of all possible codewords $\boldsymbol{x}$ is a nonbinary code of length $N$. As the mapping $\Phi$ is one-to-one, an alternative binary code $\mathcal{C}$ of length $m N$ can be constructed. At the transmitter, a binary codeword $\boldsymbol{b} \in \mathcal{C}$ is selected and at the receiver a length- $m N$ vector of L-values $\boldsymbol{l}$ is calculated. With a slight abuse of notation, we write $b_{j, i}$ and $l_{j, i}$ to denote the $j$ th input bit to the modulator and the $j$ th L-value from the demapper in the $i$ th channel use, respectively. In the length- $m N$ vector $\boldsymbol{b}$ (and similarly for $\boldsymbol{l}$ ) $b_{j, i}$ corresponds to the entry $b_{N(j-1)+i}$. This way, all input bits that correspond to a particular bit position appear consecutively in $\boldsymbol{b}$, i.e., $\boldsymbol{b}=\left[\ldots, b_{j, 1}, b_{j, 2}, \ldots, b_{j, N}, b_{j+1,1}, \ldots\right]$.

The standard BICM decoder we consider in this paper is defined as [8, eq. (3)]

$$
\hat{\boldsymbol{b}}=\underset{\boldsymbol{b} \in \mathcal{C}}{\operatorname{argmax}} \sum_{i=1}^{N} \sum_{j=1}^{m} b_{j, i} l_{j, i}
$$

i.e., the decoder finds the codeword that maximizes the correlation with the vector of the observed L-values. The codeword error probability is defined as $p_{\mathrm{e}}=\operatorname{Pr}(\hat{\boldsymbol{B}} \neq \boldsymbol{B})$.

To simplify the notation, one of the indices $i, j$ may be omitted depending on the discussed context. To avoid ambiguity, in the rest of the paper, the following convention applies: the index $j \in[m]$ is used to indicate the bit position and the index $i \in[N]$ is used to indicate the time instant.

\section{Bit-LEVEL ANALYSIS}

When analyzing achievable rates of BICM, it is common to proceed with a parallel independent channel model and assume that there exist $m$ independent bit channels from $B_{j}$ to $L_{j}$. This assumption is typically motivated by the insertion of the so-called "ideal interleaver" [7, Sec. II-B]. In this paper, we use a different approach which does not rely on any interleaver or independence assumption between bit channels. We reduce the $m$-input $m$-output channel in Fig. 1 to a channel with only one binary input and one continuous output. This can be done by specifying a behavioral model for the other, unused bit positions. To that end, consider the hypothetical scenario where we are only interested in transmitting data from $B_{j}$ to $L_{j}$. To do so, we may feed the modulator at all other bit positions $j^{\prime} \neq j$ with independent uniformly distributed (i.u.d.) bits. If the i.u.d. condition is not satisfied, the symbols are not equiprobable and the system model assumptions in Section $\llbracket-\mathrm{A}$ are violated. At the receiver side, we discard all L-values except the one of interest. This 
is conceptually shown in Fig. 4 for $j=1$ leading to a binaryinput, continuous-output channel from $B_{1}$ to $L_{1}$.

Since the results in this section are not dependent on any particular $j$, the bit-level index $j$ is dropped. Definitions and equations that hold for both exact and max-log L-values will be stated with the generic placeholder variable $L$. As an example, the generic bit-level channel in Fig. 4 is denoted by $f_{L \mid B}(\cdot \mid \cdot)$. For exact L-values, the channel is then denoted by $f_{L^{\mathrm{e}} \mid B}(\cdot \mid \cdot)$. Note that this conditional PDF is hard to calculate in general [2, Ch. 4]. For max-log L-values, the channel is denoted by $f_{L^{m \mid} \mid B}(\cdot \mid \cdot)$. This conditional PDF is relatively easy to obtain due to the special form of (4) and corresponds to a summation of piecewise Gaussian functions (see [13] and references therein).

\section{A. Bit-Level Coding Scheme}

The coding scheme for the bit-level channel in Fig. 4 is obtained from the one described in Section $\amalg$ II-D by simply omitting irrelevant bit positions. Let $\mathcal{C} \subset \mathcal{B}^{N}$ denote a binary code of length $N$ and rate $R=\log _{2}(|\mathcal{C}|) / N$. The decoder in (5) then reduces to

$$
\hat{\boldsymbol{b}}=\underset{\boldsymbol{b} \in \mathcal{C}}{\operatorname{argmax}} \sum_{i=1}^{N} b_{i} l_{i}
$$

where the $l_{i}$ are either exact or max-log L-values.

\section{B. Achievable Rates}

1) Generalized Mutual Information: The maximum achievable rate for the decoder in (6) is lowerbounded by [19. eq. (25) 1] (see also [10, eq. (18)])

$$
\mathrm{GMI}_{L}=1-\inf _{s \geq 0} \mathrm{E}\left\{\log _{2}\left(1+e^{-s \check{B} L}\right)\right\}
$$

which was originally introduced in [20] for discrete memoryless channels. We refer to this quantity as the bit-level GMI or simply GMI. The GMI has the following operational meaning. There exists a binary code $\mathcal{C}$ with rate arbitrarily close to $\mathrm{GMI}_{L}$ that can achieve reliable communication (i.e., $p_{\mathrm{e}}<\varepsilon$ for $\varepsilon$ as small as desired) as $N \rightarrow \infty$ over the channel from $B$ to $L$ assuming the decoder in (6). The codewords of such a code $\mathcal{C}$ are composed of i.u.d. bits [19] and such codes are called i.u.d. codes.

2) Mutual Information: Lifting the decoder assumption, the largest achievable rate for the channel in Fig. 4 is given by the MI between $B$ and $L$ defined as [21, p. 251]

$$
\mathrm{MI}_{L}=I(B ; L)=\mathrm{E}\left\{\log _{2}\left(\frac{f_{L \mid B}(L \mid B)}{f_{L}(L)}\right)\right\} .
$$

The MI has a similar operational meaning as the GMI, but does not make any restrictions regarding the decoder structure. In particular, for the channel from $B$ to $L$, there exists a binary code $\mathcal{C}$ with rate arbitrarily close to $\mathrm{MI}_{L}$ that can achieve reliable communication as $N \rightarrow \infty$. Furthermore, the $\mathrm{MI}$ is

${ }^{1}$ To obtain 7 from [19] eq. (25)], the decoding metric in [19] is chosen as $d(\check{B}, L)=-\check{B} L$ together with $a(\check{B})=0$, where the minus sign comes from the fact that the metric in [19] is minimized, whereas it is maximized in 6. the maximum achievable rate. Note that both the GMI and the MI are functions of the SNR. However, to simplify the notation, we omitted the dependence on $\rho$.

\section{L-values}

1) Exact L-values: For exact L-values, the decoder (6) corresponds to the maximum-likelihood decoder for the channel $f_{L^{\mathrm{ex}} \mid B}(\cdot \mid \cdot)$. This explains that for exact L-values, the GMI is equivalent to the MI. In fact, the MI for exact L-values can alternatively be written as

$$
\mathrm{MI}_{L^{\mathrm{ex}}}=I\left(B ; L^{\mathrm{ex}}\right)=I(B ; Y)=\mathrm{GMI}_{L^{\mathrm{ex}}}
$$

and the infimum in (7) is achieved for $s=1$, as shown in [8, Cor. 1].

2) Max-Log L-values: For max-log L-values, the MI is given by

$$
\mathrm{MI}_{L^{\mathrm{ml}}}=I\left(B ; L^{\mathrm{ml}}\right) .
$$

Unlike for exact L-values, $\mathrm{MI}_{L^{\mathrm{ml}}} \geq \mathrm{GMI}_{L^{\mathrm{ml}}}$. This is because max-log L-values are not true log-likelihood ratios for the channel $f_{L^{\mathrm{ml}} \mid B}(\cdot \mid \cdot)$ and hence, the decoder in (6) does not correspond to a maximum-likelihood decoder. However, applying different functions to $L^{\mathrm{ml}}$ may increase the corresponding GMI, which is in sharp contrast to the MI and the data processing inequality [21, Th. 2.8.1]. In [9, Th. 1] (see also [3, Th. 7.5]), it is shown that $\mathrm{MI}_{L^{\mathrm{ml}}}=\mathrm{GMI}_{g\left(L^{\mathrm{ml}}\right)}$ for

$$
g(l)=\log \left(\frac{f_{L^{\mathrm{m} \mid} \mid B}(l \mid 1)}{f_{L^{\mathrm{m} \mid} \mid B}(l \mid 0)}\right) .
$$

The intuitive interpretation is that the processing in (11) matches the metrics to the decoder (6), and hence, makes the decoder a maximum-likelihood decoder.

We can compare the discussed achievable rates in the form of the following chain of inequalities

$$
\mathrm{GMI}_{L^{\mathrm{ex}}}=\mathrm{MI}_{L^{\mathrm{ex}}} \stackrel{(a)}{\geq} \mathrm{MI}_{L^{\mathrm{ml}}}=\mathrm{GMI}_{g\left(L^{\mathrm{ml}}\right)} \geq \mathrm{GMI}_{L^{\mathrm{ml}}}
$$

where inequality (a) follows from the data processing inequality. As mentioned in Section [I it is commonly assumed that inequality (a) is strict. In the next section, we show that this inequality is in fact an equality in some cases.

\section{Lossless Max-Log Approximation}

We start with the following lemma.

Lemma 2. For any one-dimensional constellation and any pattern, $\mathrm{MI}_{L^{\mathrm{ex}}}=\mathrm{MI}_{L^{\mathrm{ml}}}$ if and only if there exists a function $f(\cdot)$ such that $L^{\mathrm{ex}}=f\left(L^{\mathrm{ml}}\right)$.

Proof: The "if" part follows from the data processing inequality. The "only if" follows from the fact that exact Lvalues form a minimal sufficient statistic for guessing $B$ based on $Y$. A minimal sufficient statistic is a function of every other sufficient statistic. In particular, assume $l^{\mathrm{ml}}(y)=l^{\mathrm{ml}}\left(y^{\prime}\right)$ for two channel observations $y$ and $y^{\prime}$. If $\mathrm{MI}_{L^{\mathrm{ml}}}=\mathrm{MI}_{L^{\mathrm{ex}}}$ (and hence max-log L-values also form a sufficient statistic), it follows from Fisher's factorization theorem [22, Ch. 22.3] that $f_{Y \mid B}(y \mid b) / f_{Y \mid B}\left(y^{\prime} \mid b\right)$ is independent of $b$, which implies 
$l^{\mathrm{ex}}(y)=l^{\mathrm{ex}}\left(y^{\prime}\right)$. Thus, there has to exist a function $f(\cdot)$ such that $L^{\mathrm{ex}}=f\left(L^{\mathrm{ml}}\right)$.

Based on this lemma, we have the following theorem.

Theorem 1. For one-dimensional M-PAM constellations, there exist only two cases for which the max-log approximation is information lossless, i.e., $\mathrm{MI}_{L^{\mathrm{ex}}}=\mathrm{MI}_{L^{\mathrm{ml}}}$. Either the pattern is equivalent to $\boldsymbol{p}_{\mathrm{I}}=\left[\mathbf{0}_{M / 2}, \mathbf{1}_{M / 2}\right]$, in which case the constellation can be arbitrary, or the pattern is equivalent to $p_{\mathrm{II}}=\left[\mathbf{0}_{M / 4}, \mathbf{1}_{M / 2}, \mathbf{0}_{M / 4}\right]$ and the constellation is symmetric.

Proof: The proof is given in Appendix B.

In practice, Theorem 1 implies that, for the two lossless cases, "full" information can be extracted from max-log Lvalues if proper processing is applied, i.e., in the form of the correction function (11). In addition to the patterns in Theorem 11, we denote the pattern $\boldsymbol{p}=[0,1,0,1, \ldots, 0,1]$ by $\boldsymbol{p}_{\mathrm{III}}$. As an example, for 4-PAM, there exist only three patterns that are not equivalent, i.e., $\boldsymbol{p}_{\mathrm{I}}=[0,0,1,1], \boldsymbol{p}_{\mathrm{II}}=[0,1,1,0]$, and $\boldsymbol{p}_{\mathrm{III}}=[0,1,0,1]$. For a symmetric 4 -PAM constellation, the first two patterns are lossless according to Theorem 1 and they correspond to the first and the second bit position in the BRGC, respectively. For the NBC, the first and second bit positions correspond to the patterns $\boldsymbol{p}_{\mathrm{I}}$ and $\boldsymbol{p}_{\mathrm{III}}$, respectively (see Fig. 2). From Theorem 1, the first bit position is again information lossless (even if the constellation is not symmetric) while the second one is not. In fact, we immediately have the following corollary.

Corollary 1. Among all possible combinations of onedimensional M-PAM constellations and labelings, a symmetric 4-PAM constellation with the BRGC (or any equivalent labeling) is the only case where all bit positions are information lossless.

Proof: It is easy to show that $\boldsymbol{p}_{\mathrm{I}}$ and $\boldsymbol{p}_{\mathrm{II}}$ or their equivalent patterns cannot be used twice in a labeling. This means that any labeling with more than two bit positions will contain a bit pattern for which the max-log approximation causes an information loss.

It is interesting to look at the function (11) and compare it with the curve obtained by plotting $l^{\mathrm{ex}}$ versus $l^{\mathrm{ml}}$, as shown in Fig. 5 for the three non-equivalent patterns $p_{\mathrm{I}}$ (red), $p_{\mathrm{II}}$ (green), and $p_{\text {III }}$ (blue). In general, for the lossless patterns, this function coincides with the curve $l^{\mathrm{ex}}$ versus $l^{\mathrm{ml}}$ and for lossy patterns it does not. The information loss then comes from the region where $g(\cdot)$ cannot recover the exact L-value.

\section{BICM ANALYSIS}

In this section, we return from the bit-level viewpoint to the original $m$-input $m$-output channel shown in Fig. 1]

\section{A. BICM Mutual Information}

The BICM MI is defined as

$$
\mathrm{MI}_{\boldsymbol{L}}^{\mathrm{bicm}}=\sum_{j=1}^{m} \mathrm{MI}_{L_{j}}=\sum_{j=1}^{m} I\left(B_{j} ; L_{j}\right)
$$

i.e., the sum of $m$ bit-level MIs, for both exact (cf. [7, eq. (15)]) and max-log L-values. Under the parallel independent

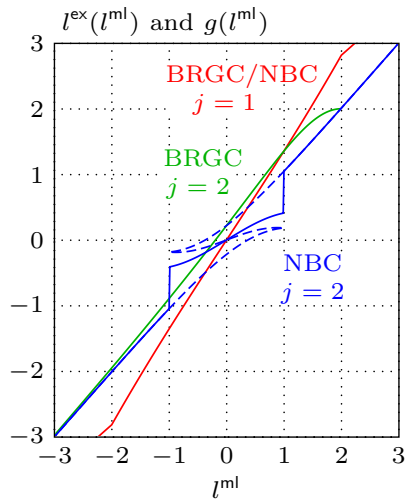

(a) $\rho=0 \mathrm{~dB}$.

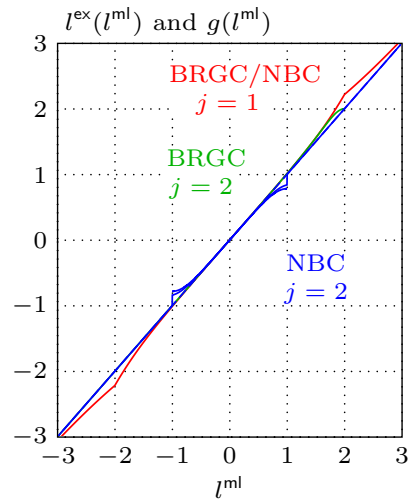

(b) $\rho=6 \mathrm{~dB}$.
Fig. 5: The correction function $g(\cdot)$ (solid) and the exact L-value versus the max-log L-value (dashed) for the three nonequivalent patterns for an equally spaced 4-PAM constellation. The values on the $\mathrm{x}$ - and $\mathrm{y}$-axes are normalized by $4 \rho / 5$.

channel model assumption [7], it is the maximum achievable rate for exact L-values with the standard BICM decoder (5). However, in the case of the model in Fig. 1, its operational meaning as an upper bound on the achievable rate is unclear. Using the mismatched decoding framework, it was shown to be an achievable rate for the standard BICM decoder [8, Sec. III]. It is also achievable for max-log L-values, provided that the ideal correction function $g(\cdot)$ is applied for each bit level before decoding via (5).

\section{B. BICM Generalized Mutual Information}

The BICM GMI for i.u.d. input bits can be written as $[8$. eq. (62)]

$$
\mathrm{GMI}_{\boldsymbol{L}}^{\mathrm{bicm}}=m-\inf _{s \geq 0} \sum_{j=1}^{m} \mathrm{E}\left\{\log _{2}\left(1+e^{-s \check{B}_{j} L_{j}}\right)\right\}
$$

and was shown to be an achievable rate for the decoder in (5] [8, Sec. III]. For exact L-values, similarly to the bitlevel GMI in (7) (see also (9)), the value $s=1$ maximizes the BICM GMI in (14). In that case, the BICM GMI can be written as a sum of bit-level GMIs. This, however, does not hold for max-log L-values.

It has recently been shown in [10] that the so-called "harmonized" GMI defined as [10, eqs. (18), (21)]

$$
\begin{aligned}
\mathrm{GMI}_{L}^{\text {harm }} & =\sum_{j=1}^{m} \mathrm{GMI}_{L_{j}} \\
& =m-\sum_{j=1}^{m} \inf _{s_{j} \geq 0} \mathrm{E}\left\{\log _{2}\left(1+e^{-s_{j} \breve{B}_{j} L_{j}}\right)\right\}
\end{aligned}
$$

is achievable when max-log L-values are used and different linear corrections are applied to L-values at different bit levels. Note that, unlike the BICM GMI, the harmonized GMI in (15) corresponds to the sum of $m$ bit-level GMIs for both exact and max-log L-values. We show in the following theorem that the harmonized GMI is achievable by the standard BICM decoder without the assumption of any L-value correction. 
Theorem 2. For any one-dimensional constellation and any labeling, the rate $\mathrm{GMI}_{L}^{\mathrm{harm}}$ in (15) is achievable by the standard BICM decoder (5).

\section{Proof: The proof is given in Appendix C}

Remark 3. The BICM GMI (14) is the largest rate for which the average error probability, averaged over all messages and i.u.d. codes, vanishes. On the other hand, the proof of Theorem 2 relies on codes that are constructed as the Cartesian product of $m$ i.u.d. codes and, hence, the overall code is not i.u.d. The fact that rates larger than those given by the GMI can be achieved with non-i.u.d. codes and mismatched decoders has also been observed in [23].

\section{Inequalities}

To clarify the difference between the achievable rates for exact and max-log L-values discussed in this section, we give a short summary in the form of the following inequalities. For exact L-values, all studied quantities are the same and we have

$$
\mathrm{MI}_{L^{\mathrm{ex}}}^{\mathrm{bicm}}=\mathrm{GMI}_{L^{\mathrm{ex}}}^{\text {harm }}=\mathrm{GMI}_{L^{\mathrm{ex}}}^{\text {bicm }}
$$

which is the rate that is achievable by the standard BICM decoder.

The value in (16) is an upper bound on the corresponding quantities for max-log L-values, i.e.,

$$
\mathrm{MI}_{L^{\mathrm{ex}}}^{\text {bicm }} \geq \mathrm{MI}_{L^{\mathrm{ml}}}^{\mathrm{bicm}} \geq \mathrm{GMI}_{L^{\mathrm{ml}}}^{\text {harm }} \geq \mathrm{GMI}_{L^{\mathrm{ml}}}^{\text {bicm }}
$$

As previously mentioned, the second quantity is an achievable rate if the function 111 is applied to the max-log L-values from the $m$ different bit positions before passing them to the decoder (5). The third quantity is a rate achievable by the standard BICM decoder (5) without any L-value correction. The last quantity corresponds to the BICM GMI as defined in [8, eq. (59)] for max-log L-values. As shown in Corollary 1 for one-dimensional constellations the first inequality is an equality only for a symmetric 4-PAM constellation labeled with a binary labeling equivalent to the BRGC.

\section{L-VALUE PROCESSING}

\section{A. Symmetrization}

In this subsection, we study how bit-level channel symmetrization affects the GMI and the MI for exact and max$\log$ L-values. Bit-level symmetrization can be motivated as follows. A binary input channel $f_{L \mid B}(\cdot \mid \cdot)$ is said to be outputsymmetric if

$$
f_{L \mid B}(l \mid b)=f_{L \mid B}(-l \mid \bar{b}) .
$$

For some patterns, for instance for $\boldsymbol{p}=[0,1,1,0]$, the channel is not output-symmetric (neither for exact nor max-log Lvalues), which complicates the analysis of BICM systems in certain cases. For example, one cannot assume the transmission of the all-zero codeword when studying the error probability $p_{\mathrm{e}}$ of a linear code over such a channel.

To enforce an output symmetric channel, it was proposed in [7] to use a randomly complemented labeling. In [24], a similar symmetrization technique was realized by the use of

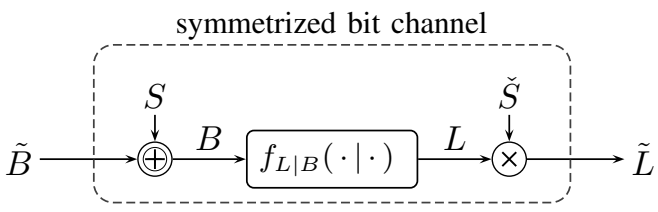

Fig. 6: Illustration of the channel symmetrization technique via i.u.d. bit adapters. $S$ is assumed to be known by both the transmitter and receiver and added to $B$ modulo 2 at the transmitter.

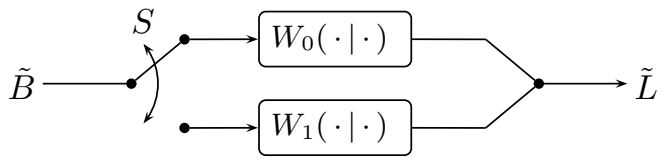

Fig. 7: Equivalent model for the channel symmetrization where $S$ is interpreted as a switch and we have $W_{0}(l \mid b)=f_{L \mid B}(l \mid b)$ (for $S=0$ ) and $W_{1}(l \mid b)=f_{L \mid B}(-l \mid \bar{b})$ (for $S=1$ ).

random independent identically distributed (i.i.d.) bit adapters as shown in Fig. 6 At the transmitter, a uniformly random bit $S$ is added modulo 2 to the transmitted bit and at the receiver, the L-value is multiplied by $\check{S}=(-1)^{S}$. The system can be thought of in the following way. The value of the adapter $S$ is known at both the transmitter and the receiver side, however, it is not known to the encoder and decoder 2 Hence, the adapter can be considered part of the channel. If we denote the symmetrized L-value by $\tilde{L}$, the conditional PDF of $\tilde{L}$ can be related to the conditional PDF of the original L-value through

$$
f_{\tilde{L} \mid \tilde{B}}(l \mid b)=\frac{1}{2}\left(f_{L \mid B}(l \mid b)+f_{L \mid B}(-l \mid \bar{b})\right) .
$$

In [24, Th. 2], it was shown that the MI is unchanged by the symmetrization if exact L-values are used. Somewhat surprisingly, the effect of this operation on the MI and the GMI for max-log L-values has not been studied in the literature. In the following, we show that the GMI is not affected by the symmetrization, while the MI is reduced for max-log L-values.

Theorem 3. For any one-dimensional constellation and any pattern, the bit-level channel symmetrization does not change the GMI in (7), i.e., $\mathrm{GMI}_{\tilde{L}}=\mathrm{GMI}_{L}$.

Proof: Let $h(x)=\log _{2}\left(1+e^{-x}\right)$. The expectation in (7) with respect to $\tilde{L}$ can then be written as

$$
\begin{aligned}
& \mathrm{E}\{h(s \tilde{\tilde{B}} \tilde{L})\} \\
& =\frac{1}{2} \sum_{b \in \mathcal{B}} \int_{-\infty}^{\infty} f_{\tilde{L} \mid \tilde{B}}(l \mid b) h(s \check{b} l) \mathrm{d} l \\
& \stackrel{(19)}{=} \frac{1}{4} \sum_{b \in \mathcal{B}} \int_{-\infty}^{\infty}\left(f_{L \mid B}(l \mid b)+f_{L \mid B}(-l \mid \bar{b})\right) h(s \check{b} l) \mathrm{d} l \\
& =\frac{1}{2} \sum_{b \in \mathcal{B}} \int_{-\infty}^{\infty} f_{L \mid B}(l \mid b) h(s \check{b} l) \mathrm{d} l \\
& =\mathrm{E}\{h(s \check{B} L)\} .
\end{aligned}
$$

\footnotetext{
${ }^{2}$ The considered system model is equivalent to the one in Section [II if $S$ is known to the decoder.
} 
Intuitively, this result can be explained by the fact that the decoder (6) does not exploit the information about the asymmetry of the L-values even if it is available.

The effect of the channel symmetrization on the mutual information is described in the following corollary.

Corollary 2. For any one-dimensional constellation and any pattern, the bit-level channel symmetrization does not change the MI, i.e., $\mathrm{MI}_{\tilde{L}_{\text {ex }}}=\mathrm{MI}_{L^{\mathrm{ex}}}$. Furthermore, we have $\mathrm{MI}_{\tilde{L}^{\mathrm{ml}}} \leq$ $\mathrm{MI}_{L^{\mathrm{ml}}}$ with equality if and only if the correction function in (11) is odd, i.e., $g(l)=-g(-l)$.

Proof: The corollary follows from Theorem [5 in Appendix $\mathrm{D}$ Indeed, the scrambler $S$ can be thought of as a switch between the two different channel laws $W_{0}(l \mid b)=$ $f_{L \mid B}(l \mid b)$ for $S=0$ and $W_{1}(l \mid b)=f_{L \mid B}(-l \mid \bar{b})$ for $S=1$, see Fig. 7. Observe that we have $\mathrm{MI}_{L}=I(B ; L)=I(\tilde{B} ; \tilde{L} \mid S) \geq$ $I(\tilde{B} ; \tilde{L})=\mathrm{MI}_{\tilde{L}}$. The necessary and sufficient condition for equality according to Theorem 5 is $g_{0}(l)=g_{1}(l)$, where $g_{j}(l)$ is defined in (85). This condition can be written as $g_{0}(l)=-g_{0}(-l)$ since

$$
g_{1}(l)=\log \frac{W_{1}(l \mid 1)}{W_{1}(l \mid 0)} \stackrel{(a)}{=}-\log \frac{f_{L \mid B}(-l \mid 1)}{f_{L \mid B}(-l \mid 0)} \stackrel{(b)}{=}-g_{0}(-l)
$$

where $(a)$ follows from $W_{1}(l \mid b)=f_{L \mid B}(-l \mid \bar{b})$ and $(b)$ follows from $W_{0}(l \mid b)=f_{L \mid B}(l \mid b)$. For exact $\mathrm{L}$-values, we always have $g_{0}(l)=l\left[3\right.$, Th. 3.10] which implies that $\mathrm{MI}_{\tilde{L}^{\mathrm{ex}}}=\mathrm{MI}_{L^{\mathrm{ex}}}$.

Remark 4. As mentioned above, the result that $\mathrm{MI}_{\tilde{L}^{\mathrm{ex}}}=$ $\mathrm{MI}_{L^{\mathrm{ex}}}$ was already proved in [24. Th. 2]. It also follows directly from Theorem 3 using the equivalence of the GMI and the MI for exact L-values in (9). 3

Observe that for exact L-values, we always have $\mathrm{MI}_{\tilde{L}_{\text {ex }}}=$ $\mathrm{MI}_{L^{\text {ex }}}$ regardless of whether the channel $f_{L^{\mathrm{ex}} \mid B}(\cdot \mid \cdot)$ is outputsymmetric or not. Obviously, for max-log L-values with an output-symmetric conditional PDF $f_{L^{\mathrm{m} \mid} \mid B}(\cdot \mid \cdot)$ we have $\mathrm{MI}_{\tilde{L}^{\mathrm{ml}}}=\mathrm{MI}_{L^{\mathrm{ml}}}$, since $g(l)=-g(-l)$. The numerical results presented in Section VI suggest that if $f_{L^{\mathrm{m} \mid} \mid B}(\cdot \mid \cdot)$ is not output-symmetric, the correction function does not satisfy $g(l)=-g(-l)$, and, hence, the inequality is strict. However, a proof for this observation does not seem to be straightforward.

The rate loss for symmetrized max-log L-values can be interpreted in the following way. In order to achieve $\mathrm{MI}_{L^{\mathrm{ml}}}$, the correction function $g(\cdot)$ needs to be applied to the Lvalues prior to decoding (6). Hence, the information about the asymmetry of the L-values is exploited by means of $g(\cdot)$. This information is lost after the symmetrization unless $g(l)=-g(-l)$, which causes the decrease of the mutual information.

From this analysis and Theorem 1, we conclude that the losses observed in [13, Fig. 4] and [14, Fig. 2] come partly from the L-value symmetrization for constellations larger than 16-ary quadrature amplitude modulation (QAM), whereas the loss for 16-QAM is solely due to the symmetrization and not

\footnotetext{
${ }^{3}$ As pointed out in [24 Th. 2], after symmetrization we lose an opportunity to optimize the input distribution, and therefore, the symmetrization may decrease the channel capacity. Channel capacity, however, is not studied in this paper.
}

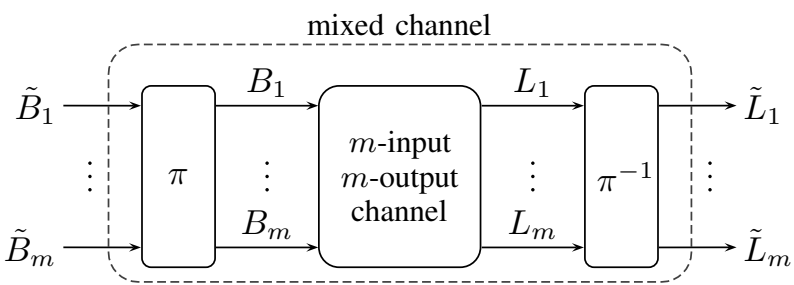

Fig. 8: Block diagram showing the mixing of bit positions. The interleaver $\pi$ reorders the bit levels.

due to the max-log approximation. This is in contrast to the discussion included in [13], [14], where the loss is attributed solely to the max-log approximation.

\section{B. Channel Mixing}

Channel mixing is another popular operation that is often assumed in order to simplify the analysis of BICM systems [25]. Channel mixing can be visualized in Fig. 8, where in addition to the $m$-input $m$-output channel, an interleaver $\pi$ and a deinterleaver $\pi^{-1}$ are introduced. The interleaver randomly and uniformly assigns the input bits to the channel inputs and the deinterleaver performs the reverse operation. Similarly to the previous section, the random assignments of the bits are known to the transmitter and the receiver. However, they are unknown to the encoder and decoder. For mixed channels, the $m$ L-values $\tilde{L}_{1}, \ldots, \tilde{L}_{m}$ from different bit positions have the same distribution and hence can be treated equally, where the PDF for all $j \in[m]$ is given by

$$
f_{\tilde{L}_{j} \mid \tilde{B}_{j}}(l \mid b)=\frac{1}{m} \sum_{j=1}^{m} f_{L_{j} \mid B_{j}}(l \mid b) .
$$

It is often said that channel mixing does not increase the BICM MI, cf. [25, Th. 2], which is obvious from the data processing inequality. In the following, we show that channel mixing does not reduce the BICM GMI either. As in the case of the bit-level symmetrization, the effect on the BICM MI depends on whether exact or max-log L-values are used.

Theorem 4. For any one-dimensional constellation and any labeling, channel mixing does not affect the BICM GMI. In fact, we have

$$
\mathrm{GMI}_{\tilde{L}}^{\text {harm }}=\mathrm{GMI}_{\tilde{\boldsymbol{L}}}^{\mathrm{bicm}}=\mathrm{GMI}_{\boldsymbol{L}}^{\mathrm{bicm}} .
$$

Proof: Using the definition of the harmonized GMI in (15) and the fact that the mixed L-values all have the same distribution we can write

$$
\begin{aligned}
\operatorname{GMI}_{\tilde{\boldsymbol{L}}}^{\text {harm }} & =m-\sum_{j=1}^{m} \inf _{s_{j}>0} \mathrm{E}\left\{\log \left(1+e^{-s_{j} \tilde{\tilde{B}}_{j} \tilde{L}_{j}}\right)\right\} \\
& =m-\inf _{s_{1}>0} \sum_{j=1}^{m} \mathrm{E}\left\{\log \left(1+e^{-s_{1} \tilde{\tilde{B}}_{j} \tilde{L}_{j}}\right)\right\}
\end{aligned}
$$




$$
\begin{aligned}
& =m-\inf _{s_{1}>0} \frac{m}{2} \sum_{b \in \mathcal{B}} \int_{-\infty}^{\infty} f_{\tilde{L}_{1} \mid \tilde{B}_{1}}(l \mid b) \log \left(1+e^{-s_{1} \breve{b} l}\right) \mathrm{d} l \\
& \stackrel{\text { 206. }}{=} m-\inf _{s_{1}>0} \frac{1}{2} \sum_{b \in \mathcal{B}} \int_{-\infty}^{\infty} \sum_{j=1}^{m} f_{L_{j} \mid B_{j}}(l \mid b) \log \left(1+e^{-s_{1} \check{b} l}\right) \mathrm{d} l \\
& =m-\inf _{s_{1}>0} \sum_{j=1}^{m} \frac{1}{2} \sum_{b \in \mathcal{B}} \int_{-\infty}^{\infty} f_{L_{j} \mid B_{j}}(l \mid b) \log \left(1+e^{-s_{1} \check{b} l}\right) \mathrm{d} l \\
& =m-\inf _{s_{1}>0} \sum_{j=1}^{m} \mathrm{E}\left\{\log \left(1+e^{-s_{1} \check{B}_{j} L_{j}}\right)\right\}
\end{aligned}
$$

where (28) proves the first equality in (27) and (29) proves the second equality.

Although channel mixing does not affect the BICM GMI, it reduces the harmonized GMI for max-log L-values, i.e., $\mathrm{GMI}_{\tilde{L}^{\mathrm{ml}}}^{\mathrm{harm}} \leq \mathrm{GMI}_{L^{\mathrm{ml}}}^{\mathrm{harm}}$ with equality if and only if all bitlevel GMIs (7) are minimized by the same value of $s$. The intuitive explanation is that the bit-level information can no longer be used by the encoder to construct $\mathcal{C}$ as a product code (see Appendix C).

Similarly to Corollary 2 the effect of channel mixing on the mutual information is given as follows.

Corollary 3. For any one-dimensional constellation and any labeling, we have $\mathrm{MI}_{\tilde{L}^{\mathrm{ex}}}^{\mathrm{bicm}}=\mathrm{MI}_{L^{\mathrm{ex}}}^{\mathrm{bicm}}$. Furthermore, we have $\mathrm{MI}_{\tilde{\boldsymbol{L}}^{\mathrm{ml}}}^{\mathrm{bicm}} \leq \mathrm{MI}_{L^{\mathrm{ml}}}^{\mathrm{bicm}}$ with equality if and only if the correction function in (11) is the same for all bit positions, i.e., $g_{j^{\prime}}(l)=$ $g_{j}(l)$ for all $j, j^{\prime} \in[m]$.

Proof: The proof is similar to the proof of Corollary 2 and follows from Theorem 5 in Appendix D

The numerical results in the next section suggest that the correction functions are different for non-equivalent patterns and, hence, the inequality is strict for all one-dimensional constellations with any labeling. However, similarly to the channel symmetrization, a proof for this observation does not seem to be straightforward.

\section{Numerical EXAMPLES}

So far, the information loss was characterized by inequalities. In this section, however, we want to compare different information rates quantitatively. To that end, we observe that all considered information rates are strictly increasing functions of the SNR. Hence, if $R=\phi(\rho)$, where $R$ is a generic information rate, then there exists $\phi^{-1}(R)=\rho$. Consider two rates $R_{1}$ and $R_{2}$ with the corresponding functions $\phi_{1}$ and $\phi_{2}$ and assume that the SNR is expressed in $\mathrm{dB}$. The loss of $R_{2}$ with respect to $R_{1}$ is defined as $\mathrm{L}\left(R_{1}\right)=\phi_{2}^{-1}\left(R_{1}\right)-\phi_{1}^{-1}\left(R_{1}\right)$. The loss $\mathrm{L}$ can be graphically interpreted as the horizontal distance between the curves $\phi_{1}(\rho)$ and $\phi_{2}(\rho)$ plotted over the SNR in $\mathrm{dB}$ for a particular value of the rate. We remark that only rates that have the same range can be compared in terms of $L$.

We first present numerical examples for the bit-level analysis to illustrate the inequalities in (12). We consider the equally spaced 4-PAM constellation with the three nonequivalent patterns defined in Section $\amalg I-D$ Fig. 9 shows the loss in dB for different achievable rates in (12) with respect to (w.r.t.) $\mathrm{MI}_{L^{\text {ex }}}$ as a function of the information rate in bits per channel use

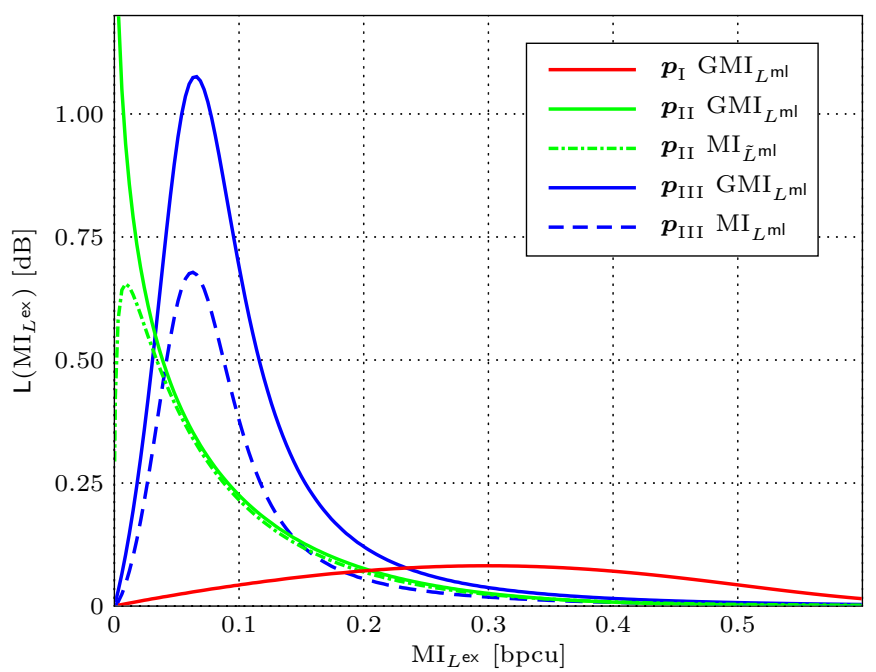

Fig. 9: Different losses for the three patterns for an equally spaced 4-PAM constellation. $\boldsymbol{p}_{\mathrm{I}}=[0,0,1,1], \boldsymbol{p}_{\mathrm{II}}=[0,1,1,0]$, and $\boldsymbol{p}_{\text {III }}=[0,1,0,1]$.

(bpcu). The solid lines show the loss for the $\mathrm{GMI}_{L^{\mathrm{ml}} \text {. It can }}$ be seen that the GMI for max-log L-values is always inferior to the MI for exact L-values. An interesting behavior of the GMI for the pattern $p_{\text {II }}$ at asymptotically low SNR is that the loss grows unboundedly when the rate goes to zero (or equivalently, when the SNR tends to zero).

The dashed line in Fig. 9 shows the loss for $\mathrm{MI}_{L^{\mathrm{ml}}}$. There is only one dashed line in the figure as two of the three patterns are information lossless. Finally, the dash-dotted line illustrates the effect of the symmetrization on the MI for the pattern $\boldsymbol{p}_{\mathrm{II}}$ and confirms Corollary 2. We also observe that the inequality in Corollary 2 in this case is strict. We remark that, for 4PAM, $p_{\text {II }}$ is the only pattern that gives such L-values (hence, only one dash-dotted curve is shown in Fig. (9).

As for the BICM analysis, we first consider an equally spaced 4-PAM constellation labeled with the NBC. Fig. 10 shows the loss for different achievable rates w.r.t. to the $\mathrm{MI}_{L^{\mathrm{ex}}}^{\mathrm{bicm}}$ as a function of the information rate. We note that the third inequality in (17) becomes an equality, i.e., $\mathrm{GMI}_{\boldsymbol{L}^{\mathrm{ml}}}^{\mathrm{harm}}=$ $\mathrm{GMI}_{L^{\mathrm{ml}}}^{\mathrm{bicm}}$, for $R \approx 0.137$. The solid lines illustrate the inequalities in (17). The red, green, and blue curves are in the order of increasing loss. The dashed red line shows the effect of channel mixing and is above the solid red line in agreement with Corollary 3 Moreover, this suggests that the inequality in Corollary 3 is strict.

In Fig. 11, we show the loss associated with the use of the max-log approximation for the 8-PAM constellation labeled with the BRGC (see Fig. 2[(c)]. The solid and dashed lines correspond to the GMI and the MI, respectively. Red, green, and blue lines show the loss for the three patterns in the BRGC, whereas the magenta lines show the loss for the entire labeling. Similarly to the GMI for $p_{\text {II }}$ in Fig. 9, the loss of the GMI for the patterns $\boldsymbol{p}_{2}$ and $\boldsymbol{p}_{3}$ goes to infinity as the rate goes to zero.

It can be seen from the figures that the losses can be quite large for low information rates (i.e., low SNR). Furthermore, the losses can even go to infinity as the rate goes to zero. 


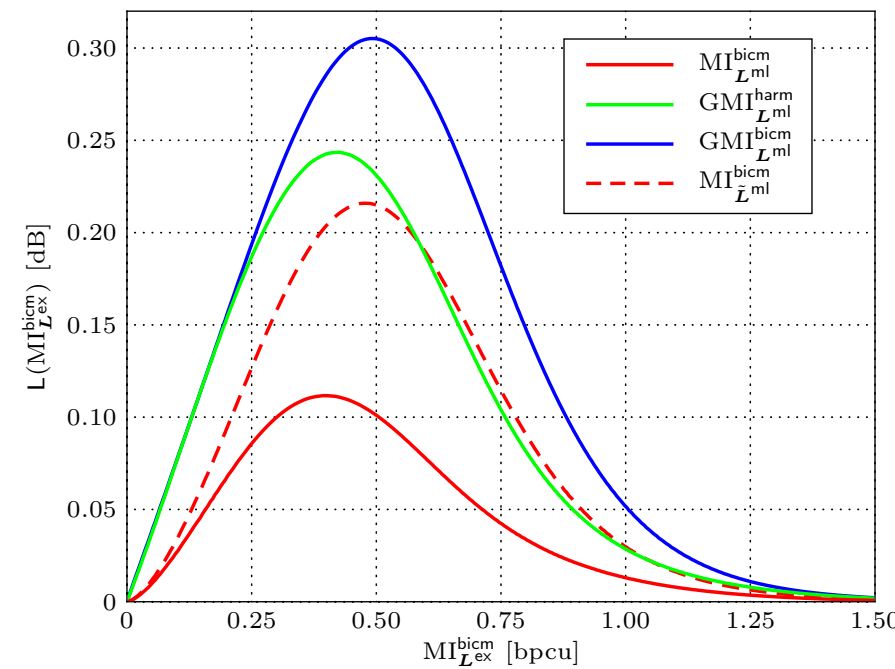

Fig. 10: Different achievable rates for 4-PAM with the NBC.

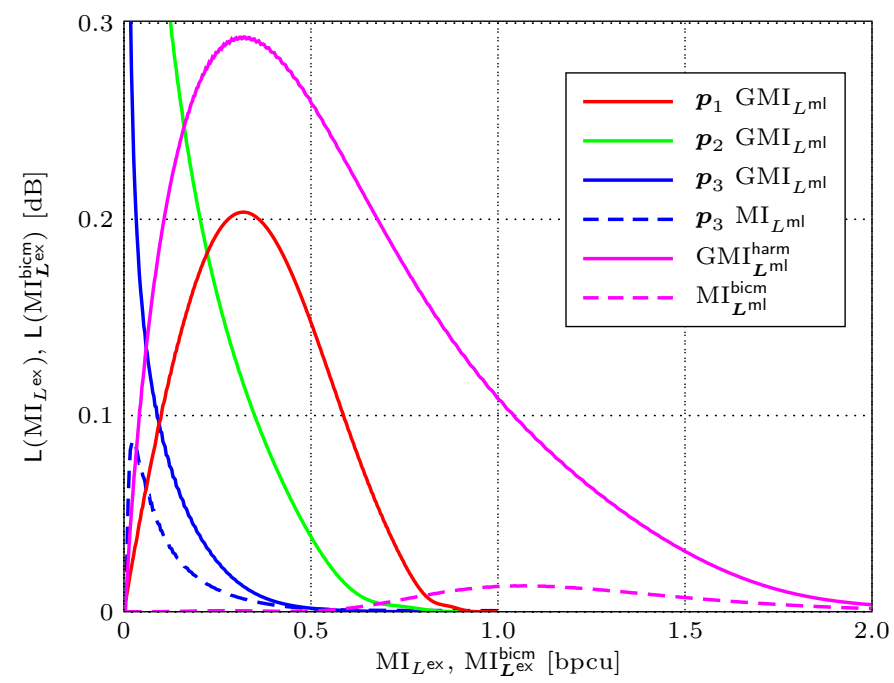

Fig. 11: Different achievable rates for 8-PAM with the BRGC. $\boldsymbol{p}_{1}=[0,0,0,0,1,1,1,1], \boldsymbol{p}_{2}=[0,0,1,1,1,1,0,0]$, and $\boldsymbol{p}_{3}=[0,1,1,0,0,1,1,0]$.

However, for moderately high rates, the losses are small. For instance, for 8-PAM with any labeling and assuming a rate of $R=m-1$ (as advised in [17] to be used in coded modulation), the loss of $\mathrm{MI}_{L^{\mathrm{ml}}}^{\text {bicm }}$ and $\mathrm{GMI}_{L^{\mathrm{ml}}}^{\text {harm }}$ w.r.t. $\mathrm{MI}_{L^{\mathrm{ex}}}^{\mathrm{bicm}}$ does not exceed $0.013 \mathrm{~dB}$ and $0.024 \mathrm{~dB}$, respectively. Therefore, we conclude that the differences between these achievable rates are negligible from a practical viewpoint.

\section{CONCLUSIONS}

In this paper, we studied achievable rates of a BICM decoder with both exact and max-log L-values for $M$-PAM constellations. We showed that the max-log approximation is not information lossy in some cases. Furthermore, when exact L-values are considered, seemingly different quantities, e.g., BICM MI, BICM GMI, or harmonized GMI are shown to give the same achievable rate. This is not the case for max$\log$ L-values as these quantities are different. For high SNR, however, the differences between them become negligible, which justifies the use of the max-log approximation in practical systems and the considered processing techniques for their analysis.

\section{APPENDIX A \\ Proof of LEMMA 1}

A symmetric constellation and pattern are easily shown to be sufficient for the exact L-value to be symmetric by using the definition of $l^{\mathrm{ex}}(y)$ in (3). To show that they are also necessary we argue as follows. Assume for a moment that the symmetry point is the origin, i.e., $y_{0}=0$. We also define $v=e^{\rho}$, where $v>1$ since $\rho>0$. We denote the subconstellation of points labeled with a 1 by $d_{1}<\cdots<d_{\tilde{M}}$ and the subconstellation of points labeled with a 0 by $t_{1}<\cdots<t_{\tilde{M}}$, where $\tilde{M}=M / 2$. Then, using (3) and $l^{\mathrm{ex}}(y)=l^{\mathrm{ex}}(-y)$, we find that, for all $y \in \mathbb{R}$,

$$
\sum_{i} v^{2 y d_{i}-d_{i}^{2}} \sum_{j} v^{-2 y t_{j}-t_{j}^{2}}=\sum_{i} v^{-2 y d_{i}-d_{i}^{2}} \sum_{j} v^{2 y t_{j}-t_{j}^{2}} .
$$

Substituting $\tilde{M}+1-i$ for $i$ in the first sum and $\tilde{M}+1-j$ for $j$ in the last sum yields

$$
\begin{aligned}
\sum_{i} v^{2 y d_{\tilde{M}+1-i}-d_{\tilde{M}+1-i}^{2}} \sum_{j} v^{-2 y t_{j}-t_{j}^{2}} \\
=\sum_{i} v^{-2 y d_{i}-d_{i}^{2}} \sum_{j} v^{2 y t_{\tilde{M}+1-j}-t_{\tilde{M}+1-j}^{2}}
\end{aligned}
$$

or, rearranging terms,

$$
\begin{aligned}
& \sum_{i, j} v^{2 y\left(d_{\tilde{M}+1-i}-t_{j}\right)-d_{\tilde{M}+1-i}^{2}-t_{j}^{2}} \\
& =\sum_{i, j} v^{2 y\left(t_{\tilde{M}+1-j}-d_{i}\right)-t_{\tilde{M}+1-j}^{2}-d_{i}^{2}} .
\end{aligned}
$$

As $y \rightarrow \infty$, the largest exponents on both sides of (32) have to be the same for the equality to hold. The largest exponents correspond to $i=j=1$ and thus

$$
v^{2 y\left(d_{\tilde{M}}-t_{1}\right)-d_{\tilde{M}}^{2}-t_{1}^{2}}=v^{2 y\left(t_{\tilde{M}}-d_{1}\right)-t_{\tilde{M}}^{2}-d_{1}^{2}}, \quad \forall y \in \mathbb{R}
$$

which yields

$$
\begin{aligned}
& d_{\tilde{M}}-t_{1}=t_{\tilde{M}}-d_{1}, \\
& d_{\tilde{M}}^{2}+t_{1}^{2}=t_{\tilde{M}}^{2}+d_{1}^{2}
\end{aligned}
$$

or equivalently

$$
\begin{aligned}
& d_{\tilde{M}}-t_{\tilde{M}}=t_{1}-d_{1}, \\
& d_{\tilde{M}}^{2}-t_{\tilde{M}}^{2}=-t_{1}^{2}+d_{1}^{2} .
\end{aligned}
$$

Factorizing [37,

$$
\left(d_{\tilde{M}}+t_{\tilde{M}}\right)\left(d_{\tilde{M}}-t_{\tilde{M}}\right)=-\left(t_{1}+d_{1}\right)\left(t_{1}-d_{1}\right) .
$$

Dividing both sides by $t_{1}-d_{1}$, which by assumption of distinct constellation points in Section $\amalg-\mathrm{A}$ is nonzero, and using 36 yields

$$
d_{\tilde{M}}+t_{\tilde{M}}=-t_{1}-d_{1} .
$$

Combining (36) with (39) yields

$$
d_{1}=-d_{\tilde{M}} \text { and } t_{1}=-t_{\tilde{M}} .
$$


We will now prove by contradiction that $d_{i}=-d_{\tilde{M}+1-i}, \forall i$. To this end, suppose the opposite, i.e., that there exists an integer $k \geq 2$ such that

$$
d_{i}=-d_{\tilde{M}+1-i}, i=1, \ldots, k-1 \text { and } d_{k} \neq-d_{\tilde{M}+1-k} .
$$

Regarding the relation between $t_{j}$ and $-t_{\tilde{M}+1-j}$, we have already proven in (40) that they are the same for $j=1$. For $j \geq 2$, we will distinguish between two cases, one of which must be true. However, both will be shown to lead to contradictions in combination with (41), which can only mean that (41) is false.

Case 1: Suppose that

$$
t_{j}=-t_{\tilde{M}+1-j}, \forall j .
$$

Then (31) and (42) together yield

$$
\sum_{i=1}^{\tilde{M}} v^{2 y d_{\tilde{M}+1-i}-d_{\tilde{M}+1-i}^{2}}=\sum_{i=1}^{\tilde{M}} v^{-2 y d_{i}-d_{i}^{2}}, \quad \forall y \in \mathbb{R} .
$$

Cancelling terms using 417,

$$
\sum_{i=k}^{\tilde{M}+1-k} v^{2 y d_{\tilde{M}+1-i}-d_{\tilde{M}+1-i}^{2}}=\sum_{i=k}^{\tilde{M}+1-k} v^{-2 y d_{i}-d_{i}^{2}}, \quad \forall y \in \mathbb{R} .
$$

Again considering $y \rightarrow \infty$, the largest exponents occur for $i=k$ on both sides. However, since $d_{k} \neq d_{\tilde{M}+1-k}$ by 441, these exponents are unequal, which contradicts (44). It can be concluded that Case 1, defined by the assumption (42), cannot be true.

Case 2: Suppose that there exists an integer $\ell \geq 2$ such that

$$
t_{j}=-t_{\tilde{M}+1-j}, j=1, \ldots, \ell-1 \text { and } t_{\ell} \neq-t_{\tilde{M}+1-\ell} \text {. }
$$

By (41) and (45), the terms in (32) for which $i<k, j<\ell$ and $i>\tilde{M}+1-k, j>\tilde{M}+1-\ell$ cancel each other. Defining $\mathcal{W} \triangleq\{(i, j): k \leq i \leq \tilde{M}+1-k$ or $\ell \leq j \leq \tilde{M}+1-\ell\}$, (32) simplifies into

$$
\begin{aligned}
& \sum_{(i, j) \in \mathcal{W}} v^{2 y\left(d_{\tilde{M}+1-i}-t_{j}\right)-d_{\tilde{M}+1-i}^{2}-t_{j}^{2}} \\
& \quad=\sum_{(i, j) \in \mathcal{W}} v^{2 y\left(t_{\tilde{M}+1-j}-d_{i}\right)-t_{\tilde{M}+1-j}^{2}-d_{i}^{2}}, \quad \forall y \in \mathbb{R} .
\end{aligned}
$$

When $y \rightarrow \infty$, the largest exponents in both sums in 46 correspond to the pairs $(i, j) \in \mathcal{W}$ for which $d_{\tilde{M}+1-i}-t_{j}$ and $t_{\tilde{M}+1-j}-d_{i}$, respectively, are maximum. Since $d_{1} \leq d_{i} \leq d_{\tilde{M}}$ and $t_{1} \leq t_{j} \leq t_{\tilde{M}}$ for all $i, j$,

$$
\begin{aligned}
& \max _{(i, j) \in \mathcal{W}} d_{\tilde{M}+1-i}-t_{j}=\max \left\{d_{\tilde{M}}-t_{\ell}, d_{\tilde{M}+1-k}-t_{1}\right\}, \\
& \max _{(i, j) \in \mathcal{W}} t_{\tilde{M}+1-j}-d_{i}=\max \left\{t_{\tilde{M}}-d_{k}, t_{\tilde{M}+1-\ell}-d_{1}\right\} .
\end{aligned}
$$

These maxima must be equal for (46) to hold for large $y$.

By (40), (41) and (45), $d_{\tilde{M}}-t_{\ell} \neq t_{\tilde{M}+1-\ell}-d_{1}$ and $t_{\tilde{M}}-$ $d_{k} \neq d_{\tilde{M}+1-k}-t_{1}$. This leaves only two possibilities to equate the right-hand sides of 477) and 487: Either

$$
d_{\tilde{M}+1-k}-t_{1}<d_{\tilde{M}}-t_{\ell}=t_{\tilde{M}}-d_{k}>t_{\tilde{M}+1-\ell}-d_{1}
$$

or

$$
d_{\tilde{M}}-t_{\ell}<d_{\tilde{M}+1-k}-t_{1}=t_{\tilde{M}+1-\ell}-d_{1}>t_{\tilde{M}}-d_{k} .
$$

Equating the dominating terms of (46) if (49) is true yields

$$
v^{2 y\left(d_{\tilde{M}}-t_{\ell}\right)-d_{\tilde{M}}^{2}-t_{\ell}^{2}}=v^{2 y\left(t_{\tilde{M}}-d_{k}\right)-t_{\tilde{M}}^{2}-d_{k}^{2}} .
$$

In analogy with (33)-(40), this equality implies $d_{k}=-d_{\tilde{M}}$, which contradicts $d_{k}>d_{1}=-d_{\tilde{M}}$. Analogously, (50) implies $d_{\tilde{M}+1-k}=-d_{1}$, which contradicts $d_{\tilde{M}+1-k}<d_{\tilde{M}}=-d_{1}$. Hence, neither (49) nor (50) can be true. It can be concluded that Case 2, defined by the assumption 445, cannot be true.

Since both Case 1 and Case 2 lead to contradictions, the assumptions (42) and (45) are both false. This proves that (41) is false, which implies $d_{i}=-d_{\tilde{M}+1-i}, \forall i$. Finally, $t_{j}=$ $-t_{\tilde{M}+1-j}, \forall j$ follows because $d_{i}$ and $t_{j}$ have equivalent roles in (30). The case $y_{0} \neq 0$ follows by applying a shift in the coordinate system.

\section{APPENDIX B \\ PROOF OF THEOREM 1}

We first recall several facts about the max-log L-value $l^{\mathrm{ml}}(y)$ in (4) and the exact L-value $l^{\mathrm{ex}}(y)$ in (3) which will be used later on in the proof.

(F1) The max-log L-value is a continuous piecewise linear function of the observation, where the slope of the linear pieces changes at the midpoints between neighboring constellation points labeled with the same bit [13].

(F2) The max-log L-value has zero-crossings at midpoints between adjacent constellation points labeled with different bits [13].

(F3) The exact L-value is an analytic function. Indeed, the nominator and the denominator in (3) are sums of exponential functions and therefore, they are analytic functions, as is their ratio. The logarithm of an analytic function is also analytic.

Note that (F2) implies that for any pattern, the max-log Lvalue has at least one zero-crossing.

The proof is structured as follows. We start by showing that for the only two patterns that induce one zero-crossing (i.e., $\boldsymbol{p}_{\mathrm{I}}=\left[\mathbf{0}_{M / 2}, \mathbf{1}_{M / 2}\right]$ and $\operatorname{inv}\left(\boldsymbol{p}_{\mathrm{I}}\right)$ ), the max-log L-value is information lossless, regardless of the constellation. We then proceed by considering patterns that induce exactly two zerocrossings. Such patterns are of the form

$$
[\underbrace{b, \ldots, b}_{a}, \underbrace{\bar{b}, \ldots, \bar{b}}_{M / 2}, \underbrace{b, \ldots, b}_{M / 2-a}]
$$

where $1 \leq a<M / 2$. We show that a necessary and sufficient condition for the max-log L-value to be information lossless is that the pattern is equivalent to $\boldsymbol{p}_{\mathrm{II}}=\left[\mathbf{0}_{M / 4}, \mathbf{1}_{M / 2}, \mathbf{0}_{M / 4}\right]$ and the constellation is symmetric. Lastly, we consider patterns that induce more than two zero-crossings and show that in this case, the max-log L-value can never be information lossless.

\section{A. One Zero-Crossing}

Consider the max-log L-value $l^{\mathrm{ml}}(y)$ in (4) for an arbitrary constellation and the pattern $\boldsymbol{p}_{\mathrm{I}}=\left[\mathbf{0}_{M / 2}, \mathbf{1}_{M / 2}\right]$. For a certain value $y$, let $a_{t}=\operatorname{argmin}_{a \in \mathcal{S}_{0}}(y-a)^{2}$ and $a_{s}=\operatorname{argmin}_{a \in \mathcal{S}_{1}}(y-a)^{2}$, where $\mathcal{S}_{0}$ and $\mathcal{S}_{1}$ are the subconstellations with points labeled with 0 and 1, respectively. 


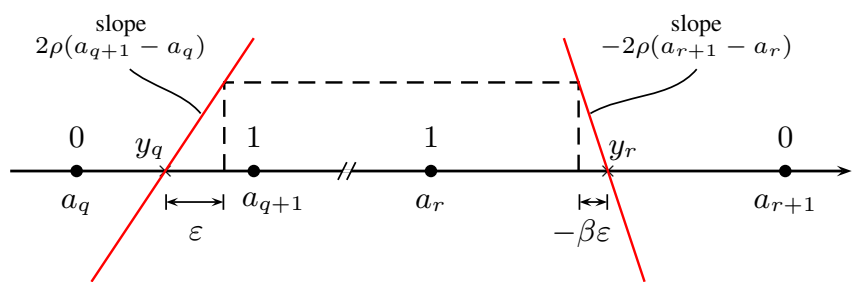

Fig. 12: Schematic representation of two neighboring zero-crossings for the max-log L-value $l^{\mathrm{ml}}(y)$ (red lines).

The max-log L-value can then be written as $l^{\mathrm{ml}}(y)=2 \rho\left(a_{s}\right.$ $\left.a_{t}\right) y+\rho\left(a_{t}^{2}-a_{s}^{2}\right)$, where $a_{s}$ and $a_{t}$ are piece-wise constant functions of $y$. Due to the structure of the pattern, $a_{s}>a_{t}$ for any value of $y$, which implies that the derivative $\mathrm{d} l^{\mathrm{ml}}(y) / \mathrm{d} y$ is positive whenever it exists. (It does not exist whenever the slope of the linear pieces changes, see (F1) above.) This, together with the fact that the max-log L-value is a continuous function of the observation, guarantees that $l^{\mathrm{ml}}(y)$ is strictly increasing. Therefore, $l^{\mathrm{ml}}(y)$ is invertible, i.e., the observation $y$ can be recovered from $l^{\mathrm{ml}}(y)$. Hence, since the exact L-value is a function of $y$, it can be obtained from the max-log L-value. The same is true for the pattern $\operatorname{refl}\left(\boldsymbol{p}_{\mathrm{I}}\right)$, in which case $l^{\mathrm{ml}}(y)$ is strictly decreasing. This claim holds for any constellation, not necessarily symmetric ones.

\section{B. Two Zero-Crossings}

Next, consider an arbitrary constellation with a pattern that induces exactly two zero-crossings, i.e., a pattern of the form in (52). Let $y_{q}$ and $y_{r}$ denote these two zero-crossings situated between the constellation points $a_{q}$ and $a_{q+1}$, and $a_{r}$ and $a_{r+1}$, respectively. This implies that $p_{q}=p_{r+1}=\bar{p}_{q+1}=\bar{p}_{r}$. Without loss of generality, we assume $p_{q}=0$. This is illustrated in Fig. 12. From the figure, we see that there exists an $\varepsilon>0$, such that

$$
l^{\mathrm{ml}}\left(y_{q}+\gamma\right)=l^{\mathrm{ml}}\left(y_{r}-\beta \gamma\right) \quad \text { for } \gamma \in[-\varepsilon,+\varepsilon]
$$

where

$$
\beta=\frac{a_{q+1}-a_{q}}{a_{r+1}-a_{r}}>0
$$

According to Lemma 2] for the max-log L-value to be information lossless, the exact L-value should be recoverable from the max-log L-value, i.e., the exact L-value has to satisfy the condition

$$
l^{\mathrm{ex}}\left(y_{q}+\gamma\right)=l^{\mathrm{ex}}\left(y_{r}-\beta \gamma\right) \quad \text { for } \gamma \in[-\varepsilon,+\varepsilon] .
$$

If this condition is not satisfied, more than one value of $l^{\text {ex }}(y)$ will correspond to one value of $l^{\mathrm{ml}}(y)$. The condition (55) can be rewritten as

$$
v(\gamma)=0 \quad \text { for } \gamma \in[-\varepsilon,+\varepsilon]
$$

where $v(\gamma)=l^{\mathrm{ex}}\left(y_{q}+\gamma\right)-l^{\mathrm{ex}}\left(y_{r}-\beta \gamma\right)$ is an analytic function (see (F3)). If an analytic function is zero on an interval, it has to be zero everywhere it is defined, i.e., $v(\gamma)=0$ for $\gamma \in \mathbb{R}$ or

$$
l^{\mathrm{ex}}\left(y_{q}+\gamma\right)=l^{\mathrm{ex}}\left(y_{r}-\beta \gamma\right) \quad \text { for } \gamma \in \mathbb{R}
$$

Using the substitution $y=\gamma-\left(y_{r}-y_{q}\right) /(1+\beta)$, we can rewrite (57) as

$$
l^{\mathrm{ex}}\left(y_{0}+y\right)=l^{\mathrm{ex}}\left(y_{0}-\beta y\right) \quad \text { for } y \in \mathbb{R} .
$$

where $y_{0}=\left(y_{r}+\beta y_{q}\right) /(1+\beta)$. We next argue that we must have $\beta=1$. Indeed, since $l^{\mathrm{ex}}(y)$ is an analytic function, it follows from (58) that

$$
l^{\mathrm{ex}(k)}\left(y_{0}+y\right)=(-\beta)^{k} l^{\mathrm{ex}(k)}\left(y_{0}-\beta y\right) .
$$

for $k \in \mathbb{N}$, where $l^{\text {ex }(k)}$ denotes the $k$ th derivative with respect to $y$. In particular, for $y=0$, we get

$$
l^{\mathrm{ex}(k)}\left(y_{0}\right)=(-\beta)^{k} l^{\mathrm{ex}(k)}\left(y_{0}\right) \quad \text { for } k \in \mathbb{N}
$$

which can only hold if either $\beta=1$ or $l^{\text {ex }(k)}\left(y_{0}\right)=0$ for all $k \in \mathbb{N}$. Assume that the latter holds. We can write $l^{\mathrm{ex}}\left(y_{0}+y\right)$ as a Taylor expansion around $y_{0}$ as

$$
l^{\mathrm{ex}}\left(y_{0}+y\right)=\sum_{k=0}^{\infty} \frac{l^{\mathrm{ex}(k)}\left(y_{0}\right)}{k !} y^{n}=l^{\mathrm{ex}}\left(y_{0}\right) .
$$

However, since $l^{\text {ex }}(y)$ cannot be constant for all $y \in \mathbb{R}$, we therefore conclude that we must have $\beta=1$, or

$$
l^{\mathrm{ex}}\left(y_{0}+y\right)=l^{\mathrm{ex}}\left(y_{0}-y\right) \quad \text { for } y \in \mathbb{R} .
$$

This means that the exact L-value has to be a symmetric function around $y_{0}=\left(y_{r}+y_{q}\right) / 2$, i.e., the midpoint between the two zero-crossings. According to Lemma 1, the constellation therefore has to be symmetric around $y_{0}$ and the pattern has to be $\boldsymbol{p}=\left[\mathbf{0}_{M / 4}, \mathbf{1}_{M / 2}, \mathbf{0}_{M / 4}\right]$ in order to satisfy $\boldsymbol{p}=\operatorname{refl}(\boldsymbol{p})$.

Consider now a symmetric constellation around $y_{0}$ and the pattern $\boldsymbol{p}_{\mathrm{II}}=\left[\mathbf{0}_{M / 4}, \mathbf{1}_{M / 2}, \mathbf{0}_{M / 4}\right]$. In this case, both the exact and the max-log L-value are symmetric functions around $y_{0}$, see Lemma 1 and Remark 2. To show that the max-log Lvalue is information lossless, it is therefore enough to show that $\left|y-y_{0}\right|$ is recoverable from $l^{\mathrm{ml}}(y)$. This can be done by showing that $l^{\mathrm{ml}}(y)$ is strictly decreasing for $y \geq y_{0}$, similarly as before.

\section{More Than Two Zero-Crossings}

Lastly, consider an arbitrary constellation $\mathcal{S}$ with a pattern such that the max-log L-value $l^{\mathrm{ml}}(y)$ has more than two zerocrossings. Now, consider any two pairs of neighboring zerocrossings and denote their (distinct) midpoints by $y_{0}$ and $y_{0}^{\prime}$, respectively. Under the assumption that the max-log L-value is information lossless and using the same arguments as above, we find that the exact L-value should satisfy both

$$
l^{\mathrm{ex}}\left(y_{0}+y\right)=l^{\mathrm{ex}}\left(y_{0}-y\right) \quad \text { for } y \in \mathbb{R}
$$

and

$$
l^{\mathrm{ex}}\left(y_{0}^{\prime}+y\right)=l^{\mathrm{ex}}\left(y_{0}^{\prime}-y\right) \quad \text { for } y \in \mathbb{R} .
$$

In the light of Remark 1, we conclude that it is not possible to satisfy both conditions simultaneously, i.e., we conclude that a max-log L-value with more than two-crossings cannot be information lossless. 


\section{APPENDIX C \\ PROOF OF THEOREM 2}

The proof follows the steps of the achievable rate analysis for multi-level coding presented in [2, Ch. 3]. In order to make the proof consistent with [2, Ch. 3], we consider a decoder that operates in the probability domain 4 according to

$$
\hat{\mathrm{m}}(\boldsymbol{y})=\underset{\mathrm{m} \in[|\mathcal{C}|]}{\operatorname{argmax}} q(\boldsymbol{b}(\mathrm{m}), \boldsymbol{y})
$$

where $\mathrm{m}$ denotes a message, $\boldsymbol{b}(\mathrm{m}) \in \mathcal{C}$ the codeword corresponding to message $\mathrm{m}$, and

$$
q(\boldsymbol{b}, \boldsymbol{y})=\prod_{i=1}^{N} \prod_{j=1}^{m} q_{j}\left(b_{i, j}, y_{i}\right)
$$

with $q_{j}\left(b_{i, j}, y_{i}\right)=\exp \left(b_{i, j} l_{j}\left(y_{i}\right)\right)$. Observe that the decoder in (65) is equivalent to the decoder in (5).

We consider an ensemble of length- $m N \operatorname{codes} \mathcal{C}$ obtained as the Cartesian product of $m$ binary codes of length $N$ according to $\mathcal{C}=\mathcal{C}_{1} \times \cdots \times \mathcal{C}_{m}$. The codewords in each code $\mathcal{C}_{j}$ are assumed to be composed of i.u.d. bits. The codewords of the code are equiprobable, the rate of the bit-level codes is given by $R_{j}=\log _{2}\left(\left|\mathcal{C}_{j}\right|\right) / N$ and the overall rate is $R=\sum_{j} R_{j}$. In the following, we only consider the case of two bit positions, i.e., $m=2$. The generalization to a larger number of bit positions is straightforward.

We let $\boldsymbol{b}_{1}\left(\mathrm{~m}_{1}\right)$ and $\boldsymbol{b}_{2}\left(\mathrm{~m}_{2}\right)$ denote the codewords in $\mathcal{C}_{1}$ and $\mathcal{C}_{2}$ corresponding to individual messages $\mathrm{m}_{1}$ and $\mathrm{m}_{2}$, respectively. When averaging over codebooks, the codewords become random vectors $\boldsymbol{B}_{1}\left(\mathrm{~m}_{1}\right)$ and $\boldsymbol{B}_{2}\left(\mathrm{~m}_{2}\right)$, where

$$
\operatorname{Pr}\left(\boldsymbol{B}_{2}\left(\mathrm{~m}_{2}\right)=\boldsymbol{b}\right)=\operatorname{Pr}\left(\boldsymbol{B}_{1}\left(\mathrm{~m}_{1}\right)=\boldsymbol{b}\right)=\prod_{i=1}^{N} p_{B}\left(b_{i}\right)
$$

for all $\mathrm{m}_{1} \in\left[\left|\mathcal{C}_{1}\right|\right]$ and $\mathrm{m}_{2} \in\left[\left|\mathcal{C}_{2}\right|\right] 5$ Due to the fact that the code is constructed as a product code, for any given codes $\mathcal{C}_{1}$ and $\mathcal{C}_{2}$ and all $\mathrm{m}_{2} \in\left[\left|\mathcal{C}_{2}\right|\right]$

$$
\begin{aligned}
\hat{\mathrm{m}}_{1}(\boldsymbol{y}) & =\underset{\mathrm{m}_{1} \in\left[\left|\mathcal{C}_{1}\right|\right]}{\operatorname{argmax}} q\left(\left[\boldsymbol{b}_{1}\left(\mathrm{~m}_{1}\right), \boldsymbol{b}_{2}\left(\mathrm{~m}_{2}\right)\right], \boldsymbol{y}\right) \\
& =\underset{\mathrm{m}_{1} \in\left[\left|\mathcal{C}_{1}\right|\right]}{\operatorname{argmax}} q_{1}\left(\boldsymbol{b}_{1}\left(m_{1}\right), \boldsymbol{y}\right)
\end{aligned}
$$

and analogously for the other bit-level code.

Since the codewords are equiprobable, the probability of error averaged over the ensemble of randomly generated codes is given by

$$
\bar{p}_{\mathrm{e}}=\frac{1}{\left|\mathcal{C}_{1}\right|\left|\mathcal{C}_{2}\right|} \sum_{\mathrm{m}_{1} \in\left[\left|\mathcal{C}_{1}\right|\right]} \sum_{\mathrm{m}_{2} \in\left[\left|\mathcal{C}_{1}\right|\right]} \bar{p}_{\mathrm{e}}\left(\mathrm{m}_{1}, \mathrm{~m}_{2}\right)
$$

where $\bar{p}_{\mathrm{e}}\left(\mathrm{m}_{1}, \mathrm{~m}_{2}\right)$ denotes the ensemble-averaged error probability conditional on messages $m_{1}$ and $m_{2}$ being transmitted. However, due to the random code construction, the probability of error is independent of the particular transmitted messages and hence $\bar{p}_{\mathrm{e}}=\bar{p}_{\mathrm{e}}\left(\mathrm{m}_{1}, \mathrm{~m}_{2}\right)$ for any given $\mathrm{m}_{1}$ and $\mathrm{m}_{2}$.

\footnotetext{
${ }^{4}$ Strictly speaking, it is the probability domain only for exact L-values.

${ }^{5}$ Even though in this paper the bits are i.u.d., i.e., $p_{B}\left(b_{i}\right)=1 / 2$, we keep the notation general to be consistent with [2] Ch. 3].
}

The probability $\bar{p}_{\mathrm{e}}\left(\mathrm{m}_{1}, \mathrm{~m}_{2}\right)$ can be calculated as

$$
\bar{p}_{\mathrm{e}}\left(\mathrm{m}_{1}, \mathrm{~m}_{2}\right)=\mathrm{E}\left\{f\left(\boldsymbol{B}_{1}\left(\mathrm{~m}_{1}\right), \boldsymbol{B}_{2}\left(\mathrm{~m}_{2}\right), \boldsymbol{Y}\right)\right\}
$$

where $f(\cdot)$ is defined in (72). For a given observation $\boldsymbol{y}$, the function $f(\cdot)$ in (72) can be upperbounded by (74), where (73) follows from the union bound and (74) follows from 69. Using again the union bound and following the standard steps of Gallager's error analysis [26], the first probability in (74) can be upperbounded as

$$
\begin{aligned}
& \operatorname{Pr}\left(\hat{\mathrm{m}}_{1}(\boldsymbol{y}) \neq \mathrm{m}_{1} \mid \boldsymbol{B}_{1}\left(\mathrm{~m}_{1}\right)=\boldsymbol{b}, \boldsymbol{Y}=\boldsymbol{y}\right) \\
& =\operatorname{Pr}\left(\bigcup_{\mathrm{m}^{\prime} \neq \mathrm{m}_{1}}\left\{\hat{\mathrm{m}}_{1}(\boldsymbol{y})=\mathrm{m}^{\prime} \mid \boldsymbol{B}_{1}\left(\mathrm{~m}_{1}\right)=\boldsymbol{b}, \boldsymbol{Y}=\boldsymbol{y}\right\}\right) \\
& \leq\left(\sum_{\mathrm{m}^{\prime} \neq \mathrm{m}_{1}} \operatorname{Pr}\left(\hat{\mathrm{m}}_{1}(\boldsymbol{y})=\mathrm{m}^{\prime} \mid \boldsymbol{B}_{1}\left(\mathrm{~m}_{1}\right)=\boldsymbol{b}, \boldsymbol{Y}=\boldsymbol{y}\right)\right)^{\gamma} \\
& =\left(\left|\mathcal{C}_{1}\right|-1\right)^{\gamma}\left(\operatorname{Pr}\left(\hat{\mathrm{m}}_{1}(\boldsymbol{y})=\mathrm{m}^{\prime} \mid \boldsymbol{B}_{1}\left(\mathrm{~m}_{1}\right)=\boldsymbol{b}, \boldsymbol{Y}=\boldsymbol{y}\right)\right)^{\gamma}
\end{aligned}
$$

for all $0 \leq \gamma \leq 1$ and any $\mathrm{m}^{\prime} \neq \mathrm{m}_{1}$. We further upperbound the probability in 76 as

$$
\begin{aligned}
\operatorname{Pr}\left(\hat{\mathrm{m}}_{1}(\boldsymbol{y})\right. & \left.=\mathrm{m}^{\prime} \mid \boldsymbol{B}_{1}\left(\mathrm{~m}_{1}\right)=\boldsymbol{b}, \boldsymbol{Y}=\boldsymbol{y}\right) \\
& =\sum_{\substack{\left.\left.\boldsymbol{b}^{\prime} \in \mathcal{B}^{N} \\
q_{1}\left(\boldsymbol{b}^{\prime}, \boldsymbol{y}\right)\right) \geq q_{1}(\boldsymbol{b}, \boldsymbol{y})\right)}} p_{\boldsymbol{B}}\left(\boldsymbol{b}^{\prime}\right) \leq \sum_{\boldsymbol{b}^{\prime} \in \mathcal{B}^{N}} p_{\boldsymbol{B}}\left(\boldsymbol{b}^{\prime}\right) \frac{q_{1}\left(\boldsymbol{b}^{\prime}, \boldsymbol{y}\right)^{s_{1}}}{q_{1}(\boldsymbol{b}, \boldsymbol{y})^{s_{1}}}
\end{aligned}
$$

where the inequality holds for any $s_{1} \geq 0$ since $\left.q_{1}\left(\boldsymbol{b}^{\prime}, \boldsymbol{y}\right)\right) \geq$ $\left.q_{1}(\boldsymbol{b}, \boldsymbol{y})\right)$ and the sum over all codewords gives an upper bound. Substituting the obtained result into (76), we have

$$
\begin{aligned}
& \operatorname{Pr}\left(\hat{\mathrm{m}}_{1}(\boldsymbol{y}) \neq \mathrm{m}_{1} \mid \boldsymbol{B}_{1}\left(\mathrm{~m}_{1}\right)=\boldsymbol{b}, \boldsymbol{Y}=\boldsymbol{y}\right) \\
& \leq\left(\left|\mathcal{C}_{1}\right|-1\right)^{\gamma}\left(\sum_{\boldsymbol{b}^{\prime} \in \mathcal{B}^{N}} p_{\boldsymbol{B}}\left(\boldsymbol{b}^{\prime}\right) \frac{q_{1}\left(\boldsymbol{b}^{\prime}, \boldsymbol{y}\right)^{s_{1}}}{q_{1}(\boldsymbol{b}, \boldsymbol{y})^{s_{1}}}\right)^{\gamma} .
\end{aligned}
$$

Averaging over all possible codewords $\boldsymbol{B}_{1}\left(\mathrm{~m}_{1}\right)$ and the observations $\boldsymbol{Y}$ gives

$$
\begin{aligned}
& \mathrm{E}\left\{\operatorname{Pr}\left(\hat{\mathrm{m}}_{1}(\boldsymbol{y}) \neq \mathrm{m}_{1} \mid \boldsymbol{B}_{1}\left(\mathrm{~m}_{1}\right)=\boldsymbol{b}, \boldsymbol{Y}=\boldsymbol{y}\right)\right\} \\
& \leq \mathrm{E}\left\{\left(\left|\mathcal{C}_{1}\right|-1\right)^{\gamma}\left(\sum_{\boldsymbol{b}^{\prime} \in \mathcal{B}^{N}} p_{\boldsymbol{B}}\left(\boldsymbol{b}^{\prime}\right) \frac{q_{1}\left(\boldsymbol{b}^{\prime}, \boldsymbol{Y}\right)^{s_{1}}}{q_{1}(\boldsymbol{B}, \boldsymbol{Y})^{s_{1}}}\right)^{\gamma}\right\} \\
& =\left(\left|\mathcal{C}_{1}\right|-1\right)^{\gamma}\left(\mathrm{E}\left\{\left(\sum_{b^{\prime} \in \mathcal{B}} p_{B}\left(b^{\prime}\right) \frac{q_{1}\left(b^{\prime}, Y\right)^{s_{1}}}{q_{1}\left(B_{1}, Y\right)^{s_{1}}}\right)^{\gamma}\right\}\right)^{N}
\end{aligned}
$$

where to go from 79 to 80 we used the fact that the channel is memoryless.

Applying similar steps to the second probability in (74) and combining (71), (74), 80), and the definition of rate for the constituent codes, we can upperbound the probability of error as

$$
\bar{p}_{\mathrm{e}} \leq 2^{-N\left(E_{1}\left(\gamma, s_{1}\right)-\gamma R_{1}\right)}+2^{-N\left(E_{2}\left(\gamma, s_{2}\right)-\gamma R_{2}\right)}
$$

where

$$
E_{j}\left(\gamma, s_{j}\right)=-\log \mathrm{E}\left\{\left(\sum_{b^{\prime} \in \mathcal{B}} p_{B}\left(b^{\prime}\right) \frac{q_{j}\left(b^{\prime}, Y\right)^{s_{j}}}{q_{j}\left(B_{j}, Y\right)^{s_{j}}}\right)^{\gamma}\right\} .
$$




$$
\begin{aligned}
& f\left(\boldsymbol{b}, \boldsymbol{b}^{\prime}, \boldsymbol{y}\right)=\operatorname{Pr}\left(\hat{\mathrm{m}}_{1}(\boldsymbol{y}) \neq \mathrm{m}_{1} \cup \hat{\mathrm{m}}_{2}(\boldsymbol{y}) \neq \mathrm{m}_{2} \mid \boldsymbol{B}_{1}\left(\mathrm{~m}_{1}\right)=\boldsymbol{b}, \boldsymbol{B}_{2}\left(\mathrm{~m}_{2}\right)=\boldsymbol{b}^{\prime}, \boldsymbol{Y}=\boldsymbol{y}\right) \\
& \leq \operatorname{Pr}\left(\hat{\mathrm{m}}_{1}(\boldsymbol{y}) \neq \mathrm{m}_{1} \mid \boldsymbol{B}_{1}\left(\mathrm{~m}_{1}\right)=\boldsymbol{b}, \boldsymbol{B}_{2}\left(\mathrm{~m}_{2}\right)=\boldsymbol{b}^{\prime}, \boldsymbol{Y}=\boldsymbol{y}\right)+\operatorname{Pr}\left(\hat{\mathrm{m}}_{2}(\boldsymbol{y}) \neq \mathrm{m}_{2} \mid \boldsymbol{B}_{1}\left(\mathrm{~m}_{1}\right)=\boldsymbol{b}, \boldsymbol{B}_{2}\left(\mathrm{~m}_{2}\right)=\boldsymbol{b}^{\prime}, \boldsymbol{Y}=\boldsymbol{y}\right) \\
& =\operatorname{Pr}\left(\hat{\mathrm{m}}_{1}(\boldsymbol{y}) \neq \mathrm{m}_{1} \mid \boldsymbol{B}_{1}\left(\mathrm{~m}_{1}\right)=\boldsymbol{b}, \boldsymbol{Y}=\boldsymbol{y}\right)+\operatorname{Pr}\left(\hat{\mathrm{m}}_{2}(\boldsymbol{y}) \neq \mathrm{m}_{2} \mid \boldsymbol{B}_{2}\left(\mathrm{~m}_{2}\right)=\boldsymbol{b}^{\prime}, \boldsymbol{Y}=\boldsymbol{y}\right) .
\end{aligned}
$$

Observe that 817 holds for any choice of $0 \leq \gamma \leq 1, s_{1} \geq 0$, and $s_{2} \geq 0$. Furthermore, the probability of error vanishes if $E_{j}\left(\gamma, s_{j}\right)>\gamma R_{j}$ for given $s_{j} \geq 0$ for $j=1,2$. In particular, if all rates satisfy

$$
\begin{aligned}
R_{j} & <\sup _{s_{j} \geq 0} \lim _{\gamma \rightarrow 0} \frac{E_{j}\left(\gamma, s_{j}\right)}{\gamma} \\
& =\mathrm{GMI}_{L_{j}}
\end{aligned}
$$

we have $\bar{p}_{\mathrm{e}} \rightarrow 0$ as $N \rightarrow \infty$. Evaluating the right-hand side of (83) by the means of derivative gives the bit-level GMI in [10, eq. (17)]. Hence, $\bar{p}_{\mathrm{e}} \rightarrow 0$ as $N \rightarrow \infty$ if $R=\sum_{j} R_{j}<$ $\sum_{j} \mathrm{GMI}_{L_{j}}$, which concludes the proof.

\section{APPENDIX D}

\section{CONVEXITY OF MUTUAL INFORMATION}

For a fixed input distribution $f_{X}(x)$, the mutual information $I(X ; Y)$ is a convex function in the channel law $f_{Y \mid X}(y \mid x)$ [21, Th. 2.7.4]. The following theorem particularizes this result to the case of binary-input, continuous-output channels and provides necessary and sufficient conditions for equality. This theorem is used in the proof of Corollaries 2 and 3 .

Theorem 5. Let $B$ be a binary $R V$ and let $S$ be a discrete $R V$ independent of $B$ taking values in $[m]$, where $m \in \mathbb{N}$. Furthermore, let $\left\{W_{j}(\cdot \mid \cdot)\right\}$, for $j \in[m]$, be a collection of binary-input, continuous-output channels with domains $\mathcal{G}_{j}=$ $\left\{l \in \mathbb{R}: W_{j}(l \mid 1) \neq 0\right.$ and $\left.W_{j}(l \mid 0) \neq 0\right\}$. Given $B=b$ and $S=s$, let $L$ be a continuous $R V$ distributed according to $W_{s}(\cdot \mid b)$. For $j \in[m]$, define the functions $g_{j}$ as

$$
g_{j}(l)=\log \left(\frac{W_{j}(l \mid 1)}{W_{j}(l \mid 0)}\right) .
$$

Then, given a fixed distribution on $B$ and $S$, we have $I(B ; L) \leq I(B ; L \mid S)$ with equality if and only if

$$
g_{j}(l)=g_{j^{\prime}}(l), \quad \text { almost everywhere on } \mathcal{G}_{j} \cap \mathcal{G}_{j^{\prime}}
$$

for all $j, j^{\prime} \in[m]$.

Proof:

We have

$$
I(B ; L \mid S)=\sum_{j=1}^{m} f_{S}(j) I(B ; L \mid S=j)
$$

where

$$
\begin{aligned}
& I(B ; L \mid S=j)= \\
& \sum_{b} f_{B}(b) \int_{\mathcal{G}_{j}} W_{j}(l \mid b) \log \left(\frac{W_{j}(l \mid b)}{\sum_{b^{\prime}} f_{B}\left(b^{\prime}\right) W_{j}\left(l \mid b^{\prime}\right)}\right) \mathrm{d} l
\end{aligned}
$$

Inserting (89) into 87) and swapping summation and integration, we obtain

$$
\begin{aligned}
& I(B ; L \mid S)= \\
& \sum_{b} f_{B}(b) \sum_{j=1}^{m} f_{S}(j) \int_{\mathcal{G}_{j}} W_{j}(l \mid b) \log \left(\frac{W_{j}(l \mid b)}{\sum_{b^{\prime}} f_{B}\left(b^{\prime}\right) W_{j}\left(l \mid b^{\prime}\right)}\right) \mathrm{d} l \\
& \geq \sum_{b} f_{B}(b) \int_{\cup_{j} \mathcal{G}_{j}} W(l \mid b) \log \left(\frac{W(l \mid b)}{\sum_{b^{\prime}} f_{B}\left(b^{\prime}\right) W\left(l \mid b^{\prime}\right)}\right) \mathrm{d} l \\
& =I(B ; L)
\end{aligned}
$$

where (92) follows from the log-sum inequality [21, Th. 2.7.1] and we defined

$$
W(l \mid b)=\sum_{j=1}^{m} f_{S}(j) W_{j}(l \mid b) .
$$

Moreover, we have equality in 92, if and only if for $b \in \mathbb{B}$

$$
\frac{W_{j}(l \mid b)}{f_{B}(0) W_{j}(l \mid 0)+f_{B}(1) W_{j}(l \mid 1)}
$$

is independent of $j \in\left\{k: l \in \mathcal{G}_{k}\right\}$ for almost all $l \in \bigcup_{j} \mathcal{G}_{j}$. This condition is equivalent to the condition that $g_{j}(l)$ for all $j \in[m]$ are equal whenever $g_{j}(l)$ are defined.

\section{REFERENCES}

[1] E. Zehavi, "8-PSK trellis codes for a Rayleigh channel," IEEE Trans. Commun., vol. 40, no. 3, pp. 927-946, May 1992.

[2] A. Guillén i Fàbregas, A. Martinez, and G. Caire, "Bit-interleaved coded modulation," Foundations and Trends in Communications and Information Theory, vol. 5, no. 1-2, pp. 1-153, 2008.

[3] L. Szczecinski and A. Alvarado, Bit-Interleaved Coded Modulation: Fundamentals, Analysis and Design. John Wiley \& Sons, 2015.

[4] IEEE 802.11, "Part 11: Wireless LAN medium access control (MAC) and physical layer (PHY) specifications," IEEE Std 802.11-2012, Tech. Rep., Mar. 2012.

[5] ETSI, "LTE; Evolved universal terrestrial radio access (E-UTRA); Physical channels and modulation," ETSI, Tech. Rep. ETSI TS 136211 V11.2.0 (2013-04), Apr. 2013.

[6] ETSI, "Digital video broadcasting (DVB); Frame structure channel coding and modulation for a second generation digital terrestrial television broadcasting system (DVB-T2)," ETSI, Tech. Rep. ETSI EN 302755 V1.3.1 (2012-04), Apr. 2012.

[7] G. Caire, G. Taricco, and E. Biglieri, "Bit-interleaved coded modulation," IEEE Trans. Inf. Theory, vol. 44, no. 3, pp. 927-946, May 1998.

[8] A. Martinez, A. Guillén i Fàbregas, G. Caire, and F. M. J. Willems, "Bit-interleaved coded modulation revisited: A mismatched decoding perspective," IEEE Trans. Inf. Theory, vol. 55, no. 6, pp. 2756-2765, June 2009.

[9] J. Jaldén, P. Fertl, and G. Matz, "On the generalized mutual information of BICM systems with approximate demodulation," in Proc. IEEE Information Theory Workshop (ITW), Jan. 2010.

[10] T. Nguyen and L. Lampe, "Bit-interleaved coded modulation with mismatched decoding metrics," IEEE Trans. Commun., vol. 59, no. 2, pp. 437-447, Feb. 2011.

[11] L. Szczecinski, "Correction of mismatched L-values in BICM receivers," IEEE Trans. Commun., vol. 60, no. 11, pp. 3198-3208, Nov. 2012. 
[12] C. Stierstorfer, "A bit-level-based approach to coded multicarrier transmission," Ph.D. dissertation, Friedrich-Alexander-Universität Erlangen-Nürnberg, Erlangen, Germany, 2009, available at http://www.opus.ub.uni-erlangen.de/opus/volltexte/2009/1395/

[13] A. Alvarado, L. Szczecinski, R. Feick, and L. Ahumada, "Distribution of L-values in Gray-mapped $M^{2}$-QAM: Closed-form approximations and applications," IEEE Trans. Commun., vol. 57, no. 7, pp. 2071-2079, July 2009.

[14] L. Szczecinski, A. Alvarado, and R. Feick, "Distribution of max$\log$ metrics for QAM-based BICM in fading channels," IEEE Trans. Commun., vol. 57, no. 9, pp. 2558-2563, Sep. 2009.

[15] E. Agrell, J. Lassing, E. G. Ström, and T. Ottosson, "On the optimality of the binary reflected Gray code," IEEE Trans. Inf. Theory, vol. 50, no. 12, pp. 3170-3182, Dec. 2004.

[16] M. Ivanov, F. Brännström, A. Alvarado, and E. Agrell, "On the exact BER of bit-wise demodulators for one-dimensional constellations," IEEE Trans. Commun., vol. 61, no. 4, pp. 1450-1459, Apr. 2013.

[17] G. Ungerboeck, "Channel coding with multilevel/phase signals," IEEE Trans. Inf. Theory, vol. IT-28, no. 1, pp. 55-67, Jan. 1982.

[18] A. J. Viterbi, "An intuitive justification and a simplified implementation of the MAP decoder for convolutional codes," IEEE J. Sel. Areas Commun., vol. 16, no. 2, pp. 260-264, Feb. 1998.
[19] A. Ganti, A. Lapidoth, and İ. E. Telatar, "Mismatched decoding revisited: General alphabets, channels with memory, and the wide-band limit," IEEE Trans. Inf. Theory, vol. 46, no. 7, pp. 2315-2328, Nov. 2000.

[20] N. Merhav, G. Kaplan, A. Lapidoth, and S. Shamai, "On information rates for mismatched decoders," IEEE Trans. Inf. Theory, vol. 40, no. 6 , pp. 1953-1967, Nov. 1994.

[21] T. M. Cover and J. A. Thomas, Elements of information theory, 2nd ed. Wiley-Interscience, 2006.

[22] A. Lapidoth, A Foundation in Digital Communication. Cambridge University Press, 2009.

[23] — "Mismatched decoding and the multiple-access channel," IEEE Trans. Inf. Theory, vol. 42, no. 5, pp. 1439-1552, Sep. 1996.

[24] J. Hou, P. H. Siegel, L. B. Milstein, and H. D. Pfister, "Capacityapproaching bandwidth-efficient coded modulation schemes based on low-density parity-check codes," IEEE Trans. Inf. Theory, vol. 49, no. 9, pp. 2141-2155, Sep. 2003.

[25] Q. Xie, Z. Wang, and Z. Yang, "Simplified soft demapper for APSK with product constellation labeling," IEEE Trans. Wireless Commun., vol. 11, no. 7, pp. 2649-2657, July 2012.

[26] R. G. Gallager, Information Theory and Reliable Communication, 1 st ed. Wiley, 1968. 